\title{
Eukaryotic translation initiation factor eIF4E-5 is required for spermiogenesis in Drosophila melanogaster
}

Lisa Shao ${ }^{1,2}$, Jaclyn M. Fingerhut ${ }^{3}$, Brook L. Falk ${ }^{1,2}$, Hong Han $^{4}$, Giovanna Maldonado ${ }^{5}$, Yuemeng Qiao ${ }^{1,6}$, Vincent Lee ${ }^{1,2}$, Elizabeth Hall ${ }^{1,2}$, Liang Chen ${ }^{4}$, Gordon Polevoy ${ }^{1}$, Greco Hernández $^{5}$, Paul Lasko ${ }^{4}$, Julie A. Brill ${ }^{1,2 \dagger}$

${ }^{1}$ Cell Biology Program, The Hospital for Sick Children, PGCRL Building, 686 Bay Street, Toronto, Ontario, M5G 0A4, Canada

${ }^{2}$ Department of Molecular Genetics, University of Toronto, 1 King's College Circle, Toronto, Ontario, M5S 1A8, Canada

${ }^{3}$ Whitehead Institute for Biomedical Research, 455 Main St, Cambridge, Massachusetts, MA 02142, USA; and Howard Hughes Medical Institute

${ }^{4}$ Department of Biology, McGill University, 3649 Promenade Sir William Osler, Montréal, Quebec, H3G 0B1, Canada

${ }^{5}$ Laboratory of Translation and Cancer, Unit of Biomedical Research on Cancer, Instituto Nacional de Cancerología (INCan), Av San Fernando 22, Mexico City, 14080, Mexico

${ }^{6}$ Human Biology Program, University of Toronto, 300 Huron Street, Toronto, Ontario, M5S 3J6, Canada

$\dagger$ To whom correspondence should be addressed:

Julie A. Brill

The Hospital for Sick Children

PGCRL Building, $15^{\text {th }}$ Floor

686 Bay Street, Room 15.9716

Toronto, Ontario M5G 0A4

CANADA

Phone: (416) 813-8863

Email: julie.brill@sickkids.ca

Running title: eIF4E-5's role in spermiogenesis

Key words: post-transcriptional regulation, eIF4E variant, spermatogenesis, individualization, spermatid cyst polarity, male fertility

Word count: 5795 


\section{Summary Statement}

46 The testis-enriched translation initiation factor eIF4E-5 is needed for spermatid cyst polarization,

47 individualization of mature sperm and male fertility in Drosophila.

\section{Abstract}

50 Drosophila sperm development is characterized by extensive post-transcriptional regulation

51 whereby thousands of transcripts are preserved for translation during later stages. A key step in

52 translation initiation is the binding of eukaryotic initiation factor 4E (eIF4E) to the 5' mRNA

53 cap. Drosophila has multiple paralogs of eIF4E, including four (eIF4E-3, -4, -5, and -7) that are

54 highly expressed in the testis. Other than eIF4E-3, none of these has been characterized

55 genetically. Here, using CRISPR/Cas9 mutagenesis, we determined that eIF4E-5 is essential for

56 male fertility. eIF4E-5 mutants exhibit defects during post-meiotic stages, including a fully

57 penetrant defect in individualization, resulting in failure to produce mature sperm. eIF4E-5

58 protein localizes to the distal ends of elongated spermatid cysts, where it regulates non-apoptotic

59 caspase activity during individualization by promoting local accumulation of the E3 ubiquitin

60 ligase inhibitor Soti. eIF4E-5 mutants also have mild defects in spermatid cyst polarization,

61 similar to mutants affecting the cytoplasmic polyadenylation-element binding protein Orb2 and

62 atypical protein kinase $\mathrm{C}(\mathrm{aPKC})$. Our results further extend the diversity of non-canonical

63 eIF4Es that carry out distinct spatiotemporal roles during spermatogenesis. 


\section{Introduction}

Translation of mRNA into protein is frequently targeted by genetic regulatory mechanisms (Kugler and Lakso, 2009; Lin and Holt, 2007; Mingle et al., 2005; Moor et al., 2017). Protein synthesis can be divided into three steps (initiation, elongation, termination), yet much of the regulation occurs at the first step (Hershey et al., 2018; Pelletier and Sonenberg, 2019; Sonenberg and Hinnebusch, 2009). During initiation, the eukaryotic initiation factor 4F (eIF4F) cap-binding complex is recruited to the 7-methylguanylate cap located at the 5' end of mRNAs. eIF4F is composed of a cap-binding protein (eIF4E) and an RNA helicase (eIF4A) held together by a scaffolding protein (eIF4G). eIF4G binds poly(A)-binding protein (PABP) and eIF3 to recruit the 40S ribosomal subunit. Translational repression can occur when eIF4Ebinding proteins (4E-BPs) bind eIF4E to block its association with eIF4G or when eIF4Ehomologous proteins (4E-HPs) bind the 5' cap to block the recruitment of eIF4E to the mRNA. Therefore, eIF4E plays an essential role in eukaryotic cap-mediated translation initiation. are not well understood. Mammals have three paralogs (eIF4E1 to eIF4E3; Joshi et al., 2004): mouse eIF4E1 and eIF4E2/4EHP mutants exhibit behavioral defects (Aguilar-Valles et al., 2018; Wiebe et al., 2020). C. elegans has five paralogs (IFE-1 to IFE-5): IFE-3, which is most similar to mammalian eIF4E1 (i.e., canonical), is essential for viability; IFE-1 is required for spermatogenesis and oocyte maturation; IFE-2 is needed for meiotic recombination; and IFE-4 is required to translate mRNAs involved in egg laying (Amiri et al., 2001; Dinkova et al., 2005; Henderson et al., 2009; Kawasaki et al., 2011; Keiper et al., 2000; Song et al., 2010). Drosophila has eight paralogs (eIF4E-1 to eIF4E-8/4E-HP) that bind to mRNA 5' caps with varying affinities in vitro (Zuberek et al., 2016). Canonical eIF4E-1 and 4E-HP are essential for viability, and eIF4E-3 is needed for meiotic chromosome segregation and cytokinesis during spermatogenesis (Brown et al., 2014; Chintapalli et al., 2007; Ghosh and Lasko, 2015;

91 Whereas $e I F 4 E-1$ and $4 E-H P$ mRNAs are ubiquitously expressed, eIF4E-3, eIF 4E-4, eIF4E-5

92 and eIF4E-7 mRNAs are highly and specifically enriched in the testis (Graveley et al., 2011).

93 This suggests that these paralogs may have distinct cellular or developmental roles during sperm 94 development. 
The stages of Drosophila male germ cell development are organized in a spatiotemporal

96

97

98

99

100

101

102

103

104

105

106

107

108

109

110

111

112 manner (Fig. S1). The stem cell niche is located at the apical tip of the testis and germ cell development progresses toward the basal end of the testis where mature sperm will eventually exit (reviewed in Fabian and Brill 2012; Fuller 1993; Lindsley and Tokuyasu, 1980; RenkawitzPohl et al., 2005). Male germline stem cells divide asymmetrically to form new stem cells and differentiating daughter cells called gonialblasts. Each gonialblast undergoes four rounds of mitosis with incomplete cytokinesis to generate a cyst of 16 primary spermatocytes. After an extended period of growth and gene expression, the 16 spermatocytes undergo meiosis with incomplete cytokinesis to form a cyst of 64 interconnected haploid spermatids. A series of dramatic morphological changes convert these round spermatids into $1.85 \mathrm{~mm}$ long mature sperm through a process called spermiogenesis. These changes include polarization of spermatid cysts relative to the long axis of the testis; assembly of flagellar axonemes that make up the sperm tails; elongation of spermatid cysts by membrane addition at the distal, growing ends; and individualization, which separates fully elongated, interconnected spermatids into individual sperm. During individualization, unneeded organelles and other cellular materials are stripped from elongated spermatid cysts by an actin-based individualization complex that moves down the length of the spermatids, forming a cystic bulge whose contents eventually pinch off into a structure called the waste bag.

Post-transcriptional regulation, possibly at the level of translation, is a crucial aspect of Drosophila spermatogenesis. Many genes needed post-meiotically are transcribed in primary spermatocytes and translationally repressed until later stages (Jayaramaiah-Raja and RenkawitzPohl, 2005; Renkawitz-Pohl et al., 2005; Santel et al., 1997; Schäfer et al., 1993; White-Cooper, 2010; White-Cooper and Caporilli, 2013; Zhao et al., 2010). In addition, a small subset of "cup" and "comet" genes, named based on their mRNA distribution, are transcribed post-meiotically, and the corresponding mRNAs localize at the growing ends of elongating spermatid cysts (Barreau et al., 2008a,b). One of these genes, soti, encodes a protein translated late in sperm development that regulates non-apoptotic caspase activity during individualization (Kaplan et al., 2010). Although little is known about how translation of cup and comet mRNAs is regulated, the cytoplasmic polyadenylation element binding protein (CPEB) Orb2 binds the 3' UTR of soti and f-cup, suggesting it might regulate their translation (Xu et al., 2012). Orb2, together with atypical 
125 protein kinase $\mathrm{C}(\mathrm{aPKC})$, ensures polarization of spermatid cysts relative to the long axis of the

126 testis (Xu et al., 2014).

127 Here, we show that the testis-enriched translation initiation factor eIF4E-5 is essential for

128 male fertility, localizes to the distal ends of elongated spermatid cysts, is required for normal

129 accumulation of Soti at the distal ends of elongated spermatids, and interacts genetically with

130 orb2 and apkc during spermatid cyst polarization. Thus, eIF4E-5 is novel player in post-

131 transcriptional regulation during spermiogenesis.

Results

eIF $4 E-5$ is required for male fertility

eIF4E-5 encodes a single predicted polypeptide of 232 amino acids that contains conserved residues needed to bind the mRNA cap (Asp108, Trp120, Glu121; Hernández et al. 2005). To examine the role of eIF4E-5 in Drosophila spermatogenesis, CRISPR/Cas9 mediated mutagenesis was used to produce $e I F 4 E-5$ alleles with deletions in the coding sequence of the gene (Fig. 1A). The deletions in $e I F 4 E-5^{B 8 a}(12 \mathrm{bp}), e I F 4 E-5^{B 8 b}(3 \mathrm{bp})$ and $e I F 4 E-5^{D 19 a}(1 \mathrm{bp})$ are predicted to produce a 4-amino acid in-frame deletion, a 1-amino acid in-frame deletion, and a frame-shift resulting in a 77-amino acid truncated protein lacking the eIF4E domain, which contains the cap-binding site (Fig. 1B). All three mutations affect the conserved motif required for binding to the eIF4E-binding motif found in eIF4Gs and other eIF4E-binding proteins (His55, Pro56, Leu57; Grüner et al., 2016): eIF4E-5 $5^{B 8 a}$ removes Pro56 and Leu57; eIF4E-5 $5^{B 8 b}$ removes His55; and $e I F 4 E-5^{D 19 a}$ has a frameshift after His55 that removes all subsequent amino acids, replacing them with 22 amino acids from an alternate reading frame. To investigate whether levels of eIF4E-5 were reduced in these mutants, polyclonal antibodies were raised against the full-length sequence of eIF4E-5. The antibodies strongly recognized a protein at the predicted molecular weight of approximately $26.9 \mathrm{kDa}$ on immunoblots of testis extracts from wild type. The level of this protein was substantially reduced in testis extracts from $e I F 4 E-5^{B 8 a}$ or $e I F 4 E-5^{B 8 b}$, and not detectable in $e I F 4 E-5^{D 19 a}$ homozygous mutants (Figs $1 \mathrm{C}$ and S2). Thus,

152 all three eIF4E-5 mutations are predicted to interfere with eIF4G binding and eIF4F complex 153 formation and result in reduced levels of eIF4E-5 protein. Males homozygous mutant for $e I F 4 E-5$ were viable and sterile, as were male $e I F 4 E-5^{B 8 a}$, 
$D f(3 L)$ Exel6279) that uncover the eIF4E-5 locus (Fig. 1D). A 1610 bp genomic rescue construct containing an N-terminal 3xFLAG in frame with the eIF4E-5 coding region restored male fertility in $e I F 4 E-5^{B 8 b}$ and $e I F 4 E-5^{D 19 a}$ homozygous mutants. These results confirm that the male sterility of eIF4E-5 mutants is due to loss of eIF4E-5 function rather than a second-site mutation generated by CRISPR/Cas9 mutagenesis. Closer inspection revealed that sperm failed to accumulate in the seminal vesicles of eIF4E-5 mutants (Fig. 1E-H) and that expression of the transgene resulted in the presence of mature, motile sperm (Fig. S3A-G). Hence, eIF4E-5 is required for male fertility.

\section{eIF4E-5 localizes to the distal ends of elongated spermatid cysts}

To begin to decipher the requirement for eIF4E-5 in male fertility, we examined eIF4E-5 protein distribution during sperm development. Immunostaining revealed that eIF4E-5 (yellow arrowheads) localized near the membrane skeletal protein Adducin (red arrowheads) at the distal ends of elongated spermatid cysts (Fig. 2A-A'). In contrast, elongated spermatid cysts from $e I F 4 E-5^{B 8 a}, e I F 4 E-5^{B 8 b}$ and $e I F 4 E-5^{D 19 a}$ homozygotes retained Adducin localization (red

172 localization of eIF4E-5, we examined the distribution of 3xFLAG-eIF4E-5 expressed from the 173 rescuing transgene. Immunostaining of testes from $3 x F L A G$-eIF $4 E-5 ; e I F 4 E-5^{D 19 a}$ males with anti-FLAG and anti-eIF4E-5 antibodies revealed that these antibodies stained the same region at the distal ends of elongated spermatid cysts (Fig. 2E-E''). Moreover, testes from eIF4E-5 $5^{\text {19a }}$

176 homozygotes lacked similar staining (Fig. 2F-F', ), confirming that the signals were specific to 177 eIF4E-5. In addition, immunostaining of testes from 3xFLAG-eIF4E-5;eIF4E-5 ${ }^{D 19 a}$ males 178 revealed that $3 \mathrm{xFLAG-eIF4E-5}$ was present in the cytoplasm of spermatocytes and elongating spermatids (Fig. 2G-G') . In contrast, testes from eIF4E-5 ${ }^{D 19 a}$ mutants revealed non-specific staining with anti-FLAG antibodies at the tip of the testis and along a subset of elongated spermatid cysts, as well as non-specific staining with anti-eIF4E-5 antibodies in the nuclei of spermatogonia, spermatocytes and spermatids (Fig. 2F-F',, $\mathrm{H}-\mathrm{H}^{\prime}$ '). Together, these results reveal that eIF4E-5 is expressed in early spermatocytes and persists through spermiogenesis, with

184 enrichment at the distal ends of elongated spermatid cysts. 
To determine the cause of male sterility in eIF4E-5 mutants, we examined testes by phase-contrast microscopy. Although overall testis morphology appeared normal, waste bags (Fig. 3A', red arrowheads) were absent, suggesting that loss of eIF4E-5 caused defects in spermatid individualization (Fig. 3A-D'). Waste bag formation was rescued by the 3xFLAGeIF4E-5 transgene (Fig. S3G,G'). Whole testes stained for activated (cleaved) caspase-3 revealed normal cystic bulges in wild-type testes (Fig. 3E,E', yellow arrowheads), whereas these appeared flattened in eIF4E-5 mutants (Fig. 3F-H', yellow arrowheads). The mutants also displayed an elevated level of active effector caspases starting at the cystic bulges and extending further towards the distal ends of the tails (Fig. 3F'-H'), as compared to wild type (Fig. 3E'). Furthermore, unlike the synchronous movement of the 64 actin-based investment cones within caspase-positive cystic bulges in wild type (Fig. 3E' 'E','), the actin cones in eIF4E-5 mutants appeared scattered and disorganized within the cystic bulges (Fig. 3F',-H','). As individualization is an essential step in the formation of mature sperm, this fully penetrant phenotype is likely the cause of male infertility in eIF4E-5 mutants.

\section{eIF4E-5 binds translational regulators 4E-BP, eIF4G-2, 4E-T and Cup}

To begin to examine the biochemical properties of eIF4E-5, we tested whether eIF4E-5 binds known eIF4E binding proteins, including eIF4G and eIF4G-2, which have roles in sperm development and male fertility (Baker and Fuller 2007; Franklin-Dumont et al., 2007; Ghosh and Lasko 2015). We performed yeast two-hybrid assays using eIF4E-5 as "bait" and constructs containing 4E-BP (Miron et al. 2001), eIF4G (Hernández et al. 1998), eIF4G-2 (Baker and Fuller 2007; Franklin-Dumont et al., 2007; Ghosh and Lasko 2015), 4E-T (Kamenska et al. 2014), Cup (Nelson et al, 2004; Zappavigna, et al., 2004) and GIGYF (Russica et al., 2019) as "prey” (Fig.

211 but not eIF4G. The lack of interaction between eIF4E-5 and eIF4G was unexpected, as we

212 previously detected a weak interaction of these proteins in a more sensitive yeast two-hybrid 213 assay (Hernández et al., 2005) and in fluorescent binding assays with a short eIF4G peptide containing the eIF4E-binding motif (Zuberek et al., 2016). In addition, unlike binding of eIF4E-5 to $4 \mathrm{E}-\mathrm{BP}, 4 \mathrm{E}-\mathrm{T}$ and Cup, binding to eIF4G-2 was sensitive to more stringent selection

216 conditions, indicating that eIF4E-5 may preferentially bind inhibitors of translation rather than 
217 translational activators. Overall, these results suggest eIF4E-5 may bind known translational

218 regulators in various complexes during sperm development.

\section{eIF4E-5 is required for localized accumulation of Soti protein}

As eIF4E-5 is essential for spermatid individualization and localizes at the distal ends of elongated spermatid cysts, we sought potential eIF4E-5 translational targets that show similar

223 localization during spermiogenesis. Among the cup and comet genes, soti encodes a testis-

224 specific E3 ubiquitin ligase inhibitor that controls caspase activation and is concentrated at the

225 distal ends of elongated spermatid cysts where its mRNA is found (Barreau et al., 2008a; Kaplan

226 et al., 2010). Because of Soti's role in individualization and similar localization of the Soti and

227 eIF4E-5 proteins, we investigated whether Soti protein distribution was affected in eIF4E-5

228 mutants. In wild type, Soti was concentrated at the distal ends of elongated spermatid cysts (Fig.

229 4A, yellow arrowheads), where it colocalized with the membrane skeletal protein Adducin (Fig.

$2304 \mathrm{D}$, red arrowheads; Hime et al., 1996). Soti protein levels were reduced in eIF4E-5 ${ }^{D 19 a}$ mutants

231 (Fig. 4B,E), and normal levels of Soti protein were rescued by expression of the 3xFLAG-eIF4E-

2325 genomic transgene (Fig. 4C, yellow arrowheads). Single molecule RNA florescent in situ

233 hybridization (smFISH) revealed that loss of eIF4E-5 did not alter soti mRNA expression or

234 localization (Fig. S5A-F). These results demonstrate that eIF4E-5 is required for localized

235 accumulation of Soti protein at the distal ends of elongated spermatid cysts.

236 Because of their similar distribution in elongated spermatid cysts, we examined whether

237 eIF4E-5 colocalizes with soti mRNA or Soti protein. smFISH of soti mRNA combined with

238 immunofluorescence of 3xFLAG-eIF4E-5 protein (Fig. 5A-A') or endogenous eIF4E-5 (Fig.

239 S5G-G') revealed that soti mRNA (cysts with dashed outlines) and eIF4E-5 protein (red

240 arrowheads) did not colocalize and were concentrated at the distal ends of different elongated

241 spermatid cysts. In addition, smFISH showed that eIF4E-5 mRNA was diffusely cytoplasmic

242 starting in primary spermatocytes and did not colocalize with soti mRNA or become

243 concentrated at the distal ends of spermatid cysts (Fig. S5H-J). In contrast, immunofluorescence

244 revealed colocalization of 3xFLAG-eIF4E-5 and Soti protein (Fig. 5B-B''), with some cysts

245 exhibiting higher (Fig. 5Bi), similar (Fig. 5Bii) or lower (Fig. 5Biii) levels of eIF4E-5 relative to

246 Soti at the distal ends the elongated spermatid cysts. These results suggest either that eIF4E-5 
promotes Soti translation concomitant with soti mRNA degradation or that eIF4E-5 affects accumulation of Soti at the distal ends of elongated spermatid cysts by an alternate mechanism.

\section{eIF4E-5 is dispensable for translation of axonemal dyneins}

Because flagellar axoneme assembly occurs at the distal ends of elongating spermatids et al., 2013; Riparbelli et al., 2012; Tokuyasu, 1975), we examined whether eIF4E-5 is needed

254 for the translation of axonemal proteins. Transcripts encoding the testis-specific axonemal dynein heavy chain K1-3 localize at the distal ends of spermatid cysts (Fingerhut and Yamashita, 2020). We used smFISH to test whether soti and $k l-3$ mRNAs colocalize in the same cysts and

257 found that although both transcripts were present at the distal ends of early elongating spermatid cysts, they did not colocalize (Fig. 6A). In addition, soti mRNA was present at the distal ends of late elongating spermatid cysts at stages when $k l-3$ mRNA was no longer enriched (Fig. 6B,C). Similar to soti mRNA, $k l-3$ mRNA (cysts with dashed outlines) did not colocalize with eIF4E-5 protein (red arrowheads) at the distal ends of elongating cysts (Fig. 6D-F). Immunoblotting of endogenously tagged K1-3 3xFLAG revealed that K1-3 protein levels were unaffected in eIF4E-5 mutants (Figs 6G and S6). Hence, soti and $k l-3$ transcript localization appears to be distinct, and the translational regulation involved in axoneme assembly is likely independent of eIF4E-5.

\section{eIF4E-5 affects polarization of spermatid cysts}

In addition to defects in individualization, eIF4E-5 mutants exhibited spermatid cysts that were mispolarized relative to the long axis of the testis (Fig. 7A-D). In wild-type testes, spermatid cysts with elongated nuclei ( $\sim 50$ cysts/testis; Zhou et al., 2011) were oriented such that all 64 nuclei within a cyst point towards the basal end of the testis and the tails point towards the

272 mispolarized relative to the long axis of the testis, such that clusters of elongated nuclei faced the 273 tip (Fig. 7B, inset, yellow arrowheads and Fig. 7D, insets; see also Fig. 2C,C', cyan

274 arrowheads). Whereas mispolarized cysts were rarely seen in wild-type testes (5\%), 30-50\% of 275 eIF4E-5 mutant testes exhibited this phenotype $(p<0.05)$, which appeared to be more common in $276 e I F 4 E-5^{B 8 b}$ mutants than eIF4E-5 $5^{B 8 a}$ mutants (Fig. 7E). The cyst mispolarization phenotype was 277 also observed but not quantified in eIF4E-5 $5^{D 19 a}$ mutants (Fig. 4B', white arrowhead). However, 
278 because nearly all spermatid cysts are oriented properly in testes from eIF4E-5 mutants, this

279 defect is likely not the cause of the observed fertility defects.

\section{eIF4E-5 acts with Orb2 and aPKC to promote spermatid cyst polarization}

The cyst polarization defect in eIF4E-5 mutants was reminiscent of phenotypes observed in mutants for the cell polarity regulator atypical protein kinase $\mathrm{C}(\mathrm{aPKC})$ and the cytoplasmic polyadenylation-element binding protein (CPEB) Orb2 (Xu et al., 2014). Heterozygosity for a null allele of either $a P K C\left(a P K C^{k 06403}\right)$ or orb2 $\left(\operatorname{orb}^{36}\right)$ leads to mispolarized spermatid cysts. To determine whether eIF4E-5 acts in the same manner as aPKC and Orb2, we examined testes from males transheterozygous for $e I F 4 E-5^{B 8 a}$ or $e I F 4 E-5^{B 8 b}$ and $a P K C^{k 06403}$ or orb $^{36}$ (Fig. 7F). In comparison to the single heterozygous mutants, which exhibited low levels of cyst mispolarization (7-17\% of testes), this phenotype was enhanced in the transheterozygotes (29$37 \%$ testes $)(p<0.05)$. These genetic results suggest that eIF4E-5 has at least a partially overlapping role with Orb2 and aPKC in controlling spermatid cyst polarization.

\section{Discussion}

\section{eIF4E-5 is required for spermiogenesis and male fertility}

Our data indicate that eIF4E-5 is essential for Drosophila male fertility and needed for faithful polarization of spermatid cysts and individualization of spermatids to form mature sperm. These post-meiotic defects in eIF4E-5 mutants are distinct from the earlier defects observed in eIF4E-3 mutants (Hernández et al., 2012), demonstrating that two of the four testisenriched eIF4Es have distinct spatiotemporal roles during Drosophila spermatogenesis. eIF4E-3 is most highly enriched in primary spermatocytes, where it is needed for meiotic chromosome segregation and cytokinesis (Hernández et al., 2012). In contrast, eIF4E-5 concentrates at the distal end of elongated spermatid cysts, a site important for regulating individualization. Although both eIF4E-3 and eIF4E-5 are transcribed in primary spermatocytes, enrichment of these eIF4E proteins coincides with the stages they regulate, supporting the idea that the evolutionary expansion of these non-canonical testis-enriched eIF4Es allows for distinct roles and regulation during spermatogenesis. Indeed, complementation experiments revealed that eIF4E-1 and eIF4E-3, but not eIF4E-5, were able to rescue growth of Saccharomyces cerevisiae lacking its only eIF4E paralog, Cdc33, suggesting that eIF4E-1/-3 and eIF4E-5 likely have 
309

310

311

312

313

314

315

316

317

318

319

320

321

322

323

324

325

326

327

328

329

330

331

332

333

334

335

336

337

338

339

different activities or require distinct binding partners in vivo. Sorting out the corresponding mechanisms will provide a better understanding of post-transcriptional regulation in Drosophila sperm development.

\section{eIF4E-5 is needed for spermatid individualization and localized Soti protein accumulation}

Non-apoptotic caspase activity is needed for progression of actin cones and degradation of unneeded organelles during individualization (Arama et al., 2003; Huh et al., 2004; Arama et al., 2007; Muro et al., 2006). In elongated spermatid cysts, caspases are initially activated at the nuclear end, where actin cones form, and repressed along the spermatid tails. As the actin cones move away from the nuclei, the peak of caspase activity remains associated with the cystic bulge. This localized caspase activity allows for controlled degradation of organelles, and disruption of this activity leads to failure of individualization, characterized by scattered actin cones. In this study, we show that localized caspase activation is disrupted and actin cones are scattered in individualizing spermatids of eIF4E-5 mutants. These results suggest that eIF4E-5 post-transcriptionally regulates non-apoptotic caspase activity during spermiogenesis.

Caspase activity is regulated during individualization by the inhibitor of apoptosis (IAP)like protein dBruce. dBruce is ubiquitinated by the cullin E3 ubiquitin ligase complex CRL3 and degraded at the nuclear end, allowing caspase activation (Arama et al., 2007). At the distal end, Soti binds and prevents CRL3 from binding dBruce and promoting its degradation (Kaplan et al., 2010). In soti mutants, CRL3 is activity leads to dBruce destruction and caspase activation along the entire length of the spermatid cysts, inhibiting proper individualization. Here, we show that localized accumulation of Soti protein is reduced at the distal end of elongated spermatids in eIF4E-5 mutants, which exhibit defects in individualization that resemble soti mutants.

Although Soti protein and eIF4E-5 colocalize at the distal ends of elongated spermatid cysts, soti mRNA and eIF4E-5 do not. One possibility is that Soti translation depends on eIF4E-5 and that soti mRNA is degraded in a co-translational manner, as seen for many transcripts in yeast and mammals (Pelechano et al., 2015; Tuck et al., 2020). However, this seems unlikely, as some Soti protein remains present in eIF4E-5 mutants. Another possibility is that one of the other testis eIF4Es might be able to translate soti, but with less efficiency than eIF4E-5, resulting in production of a smaller amount of Soti protein. Yet another explanation could be that eIF4E-5 regulates Soti translation indirectly or that it regulates other mRNA targets that are needed for 
individualization. In addition to soti, many transcripts have been identified that are postmeiotically transcribed and accumulate at the distal ends of elongating spermatid cysts in cup or comet patterns (Rathke et al., 2007; Barreau et al., 2008a,b; Vibranovski et al., 2009, 2010). It is possible that one or more of these transcripts is needed for individualization and requires eIF4E5 for its localization or localized translation. Alternatively, eIF4E-5 might promote Soti accumulation independent of any role it may have in mRNA regulation. Thus, although our results reveal a novel requirement for eIF4E-5 in promoting regulation of non-apoptotic caspase activity during Drosophila spermatogenesis, its mechanism of action remains obscure. Distinguishing among these possibilities will be the subject of future studies.

\section{eIF4E-5 acts with aPKC and Orb2 to regulate spermatid cyst polarization}

For successful transfer of mature sperm to the seminal vesicle, spermatid cysts must polarize such that nuclei face the basal end of the testis, and the tails point towards the tip. aPKC and Orb2 are involved in polarization of the cysts; heterozygous $a P K C$ or orb2 mutants exhibit bundles of 64 spermatids whose polarity is reversed relative to the long axis of the tissue (Xu et al., 2014). Orb2 ensures localization and localized translation of $a P K C$ mRNA to establish spermatid cyst polarity, and transheterozygotes of $a P K C$ and $o r b 2$ have a more severe defect than heterozygotes alone (Xu et al., 2014). Here, we show that eIF4E-5 mutants exhibit the same polarization defect as $a P K C$ and orb2 mutants, with a subset of spermatid cysts pointing towards the wrong end of the testis. Transheterozygous mutants of $e I F 4 E-5$ and $a P K C$ or orb2 have a more severe polarity defect than the heterozygous mutants alone, suggesting that eIF4E-5, aPKC and Orb2 might act in the same pathway to establish spermatid cyst orientation. aPKC protein localizes at the growing ends of elongating spermatids where its mRNA is also found (Xu et al., 2014), raising the possibility that $a P K C$ mRNA or protein accumulation might be regulated by eIF4E-5.

In addition to localized translation of $a P K C$ in spermatids, there is evidence that polarity 366 proteins are post-transcriptionally regulated in different cell contexts (Barr et al., 2016). For example, Par-3 mRNA is locally translated for axonal outgrowth in embryonic rat neurons (Hengst et al., 2009; Macara et al., 2009). This raises the possibility that polarity proteins other 369 than aPKC are similarly regulated in spermatids. Xu et al. (2014) showed that Bazooka, Dlg and 
371 of post-transcriptional regulation. However, the authors describe a subset of cysts as

372 mispolarized in par-6 heterozygotes. Thus, Par-6 might also act with eIF4E-5, aPKC and Orb2 to

373 control spermatid cyst polarization, and that its transcript could be a potential target of eIF4E-5

374 regulation. Our results add to the existing literature that post-transcriptional regulation plays an

375 important role in cyst polarization during Drosophila spermatogenesis and indicate that eIF4E-5

376 participates in this process.

\section{Regulation of eIF4E-5 during spermiogenesis}

Although the relationship of eIF4E-5 to aPKC translation is unclear, our data show that Soti translation does not rely solely on eIF4E-5. In addition, eIF4E-5 is dispensable for translation of at least one other transcript found at the distal ends of spermatid cysts, $k l-3$. Indeed, it appears that eIF4E-5 accumulates in elongated spermatids at a later stage than soti and $\mathrm{kl}-3$ mRNAs. Because K1-3 is required for construction of the flagellar axoneme during elongation, and $e I F 4 E-5$ is required for individualization at a later stage, eIF4E-5 localization to the distal end likely initiates around the time spermatids become fully elongated. Because eIF4E-5 protein is present in the cytoplasm of male germ cells in primary spermatocytes and spermatids, and its transcript also shows a diffuse localization at these stages, it appears unlikely that eIF4E-5 itself is post-transcriptionally regulated. Thus, it remains unclear how eIF4E-5 protein becomes concentrated at the distal end. Perhaps the mRNA localization mechanism involved in transporting the cup and comet transcripts from nuclei also regulates localization of eIF4E-5 protein. Alternatively, it is possible that local translation of eIF4E-5 depends on translational machinery that is present at the distal end. It will be of interest to identify the factors needed for mRNA transport and translation at the distal ends of elongated spermatid cysts.

Our results indicate that eIF4E-5 directly binds several known eIF4E binding partners (4E-BP, eIF4G-2, 4E-T, Cup) and might act in the same pathway as Orb2. As all of these proteins regulate mRNA translation (Miron et al. 2001; Kamenska et al. 2014; Nelson et al,

397 2004; Zappavignia, et al., 2004; Baker and Fuller 2007; Franklin-Dumont et al., 2007), interactions with these proteins may help facilitate or repress translational activity of eIF4E-5 and its target mRNAs at different stages of spermiogenesis. Although canonical eIF4E-1 and testis-specific eIF4E-3 both associate with canonical eIF4G and testis-specific eIF4G-2 (Ghosh 
and Lasko, 2015), eIF4E-5 shows a stronger interaction with eIF4G-2 than eIF4G. Thus, an eIF4F complex formed by eIF4E-5 and eIF4G-2 might target transcripts for translation in vivo.

\section{Conclusion}

Here, we show that the testis-specific Drosophila eIF4E paralog eIF4E-5 is essential for male fertility. Loss of eIF4E-5 disrupts localized accumulation of the caspase inhibitor Soti during individualization and hence regulated activation of Caspase-3. In addition, eIF4E-5 acts with Orb2 and aPKC to promote spermatid cyst polarization. Our study provides evidence of localized post-transcriptional regulation by eIF4E-5 during two developmental stages of Drosophila spermatogenesis. Future experiments will reveal the mechanism by which eIF4E-5 acts to promote male fertility.

Although there are apparent differences between Drosophila and human spermatogenesis, activation of pro-apoptotic proteins without causing the death of the entire cell

414 is also used to eliminate cytoplasmic components during terminal differentiation of mammalian spermatids (Shaha et al., 2010). Because one known cause of human male infertility is incomplete extrusion of cytoplasm (Rengan et al., 2012), it would be of interest to know if there

417 is a similar role for post-transcriptional regulation in promoting this aspect of male fertility in humans.

\section{Materials and Methods}

\section{Fly strains and husbandry}

Flies were raised on standard cornmeal molasses agar at $25^{\circ} \mathrm{C}$ (Ashburner, 1990). $w^{1118}$

423 was used as the experimental control. $w^{1118}$; PBac\{vas-Cas9\} (Bloomington Drosophila Stock

424 Center (BDSC) \#56552, Bloomington, USA) was used to generate eIF4E-5 CRISPR/Cas9

425 mutants. $y^{l} M\{3 x P 3-R F P-3 x P 3-G F P$-vas-int.DM $\} Z H-2 A w^{*} ; P\{C a r y P\} a t t P 40$ (BestGene Inc.)

426 contains a second chromosome attP site (25C6) and was used to generate 3xFLAG-eIF4E-5

427 transgenic lines. Double-balancer stock $w^{1118}$; Sco/CyO; MKRS/TM6B was used for balancing

428 mutants. eIF4E-5 alleles were examined in trans to chromosomal deletions Df(3L)BSC631

429 (BDSC \#25722) and Df(3L)Exel6279 (BDSC \#7745) lacking the entire eIF4E-5 coding region.

$430 K l-3^{3 x F L A G}$ carries a $3 \times F L A G$ tag at the endogenous C-terminus of the $k l-3$ coding region,

431 generated by CRISPR/Cas9 mediated knock-in (Fingerhut et al., 2019). UAS-kl-3 $3^{\text {TriP.HMC03546 }}$ 
432 (BDSC \#53317) expresses dsRNA for RNAi directed against $k l$-3 under UAS control (Perkins et 433 al., 2015). $a P K C^{k 06403}$ (BDSC \#10622) carries a $P\{$ lac $W\}$ insertion between two promoters in the 434 third intron, resulting in a loss of function allele (Xu et al., 2014). Orb2 ${ }^{36}$ (BDSC \#58479) carries

435 a deletion of the Orb2 coding region generated through FRT-mediated recombination between 436 two flanking progenitor insertions of $P B a c\{W H\} C G 43783^{f 04965}$ and $P B a c\{W H\} o r b 2^{f 01556}$ (Xu et 437 al., 2012).

438 Fertility tests were performed by mating individual males of each genotype with five 439 virgin $w^{1118}$ females at $25^{\circ} \mathrm{C}$. After 5 days, crosses were observed for the presence of progeny.

Generation of $e I F 4 E-5$ mutant strains selected using the CRISPR Optimal Target Finder (http://targetfinder.flycrispr.neuro.brown.edu;

444 Gratz et al., 2014): 5'-GAATTTTGTCGCGATTCGAG-3' (gRNA1) and 5'-

445 GAGTCGAGTACAAGCATCCTT-3' (gRNA2). The two selected gRNAs were cloned into 446 pCFD4d under two promoters, U6-1 and U6-3 (Addgene plasmid \#83954, Watertown, USA; Ge 447 et al., 2016). pCFD4d was digested with BbsI (New England Biolabs, R3539L, Waltham, USA) 448 and gel purified. Inserts were generated by PCR using the following primers and pCFD4d as a 449 template: 5'AGCTAGAAATAGCAAG-3' and 5'ATTTTAACTTGCTATTTCTAGCTCTAAAACAAGGATGCTTGTACTCGACTCGACGTTA AATTGAAAATAGGTC-3'. The backbone and insert were combined by Gibson Assembly

454 Master Mix (New England Biolabs, E2611L). The gRNA plasmid was confirmed by sequencing 455 and injected into transgenic embryos expressing Vasa-Cas9 and allowed to develop to adulthood 456 (BestGene Inc., Chino Hills, USA). Each adult fly was individually crossed with a balancer stock 457 to generate stocks of putative mutants. Genomic DNA was extracted from homozygous putative 458 mutants of each stock for PCR amplification of eIF4E-5 and sequenced using primers: 5'459 GGTGATGACACTACTGACGC-3' and 5'-AACGCCCAACAAACTGAAAC-3' (The Centre 460 for Applied Genomics, The Hospital for Sick Children, Toronto, Canada). This experiment was 461 repeated twice; the initial round identified two different mutant alleles from the same founder 
462 parent (eIF4E-5 $5^{B 8 a}$ and $\left.e I F 4 E-5^{B 8 b}\right)$ and the second round identified a frame-shift allele (eIF4E$\left.4635^{D 19 a}\right)$.

\section{Generation of genomic 3xFLAG-eIF4E-5 rescue construct and transgenic flies}

The rescue construct consisted of the genomic DNA starting 359 bp upstream of the ATG, 69 bp 3xFLAG tag (5'CGATGACAAG-3'), 12 bp linker (5'- GGCAGCGAATTC-3'), all 791 bp of eIF4E-5 protein coding sequence including introns, and $362 \mathrm{bp}$ downstream of the stop codon including the putative poly(A) site. The first three regions (5' region, 3xFLAG, linker) was synthesized

472 (BioBasic Inc., Markham, Canada) and subcloned into the pattB plasmid (Drosophila Genomics

473 Research Center (DGRC), 1420, Bloomington, USA) using BamHI (New England Biolabs,

474 R3136S) and EcoRI (New England Biolabs, R3101S). The last two regions were PCR-amplified

475 from genomic DNA with EcoRI and NotI (New England Biolabs, R3189S) added to the primers

476 (5'-ATGACAAGGGCAGCGAATTCATGGCCAGTGCACAAGTG -3'and 5'-

477 GTACCCTCGAGCCGCGGCCGCGCTTGAGTAGGCAATTACGAC-3'), and subcloned into

478 pattB downstream and in-frame with the 5' genomic region, 3xFLAG and linker. The pattB-

$4793 \times 3 F$ AG-eIF4E-5 plasmid was confirmed by sequencing and integrated into the attP40 site on

480 the second chromosome via PhiC31 integrase-mediated transgenesis by injection into $y^{l}$

$481 M\{3 x P 3-R F P-3 x P 3-G F P-v a s-i n t . D M\} Z H-2 A w^{*} ; P\{C a r y P\} a t t P 40$ embryos (BestGene Inc.).

\section{Generation of anti-eIF4E-5 antibodies}

The full-length coding region of eIF4E-5 was PCR-amplified using the plasmid 4E5pCR2.1 as a template (Hernández et al., 2005) and subcloned into pRSET (Invitrogen, V35120) to create an expression construct. The plasmid was transformed into E. coli BL21 (DE3) (Novagen, 71012) to produce a His6X fusion recombinant protein according to the manufacturer's instructions. The fusion protein was purified using Ni-NTA beads under denaturing conditions (Thermo Fisher Scientific, R90101, Waltham, USA). Polyclonal antieIF4E-5 antibodies were raised in rabbit against this recombinant protein (Comparative Medicine

491 and Animal Resources Centre, McGill University, Montreal, Canada). The sera were separated and $\mathrm{NaN}_{3}$ was added at a concentration of $0.02 \%$. 


\section{Squashed preparations of Drosophila testes}

Testes were dissected from 0 to 2-day old males (unless otherwise stated) in cold testis isolation buffer (TIB) (Casal et al., 1990). Whole testes were mounted on polylysine coated slides in TIB and squashed with a coverslip (Polysine, P4681-001, Thermo Fisher Scientific). Live images for Fig. 3 were acquired on an upright Zeiss Axioplan 2E epifluorescence microscope equipped with a 20x phase-contrast objective and an Axiocam black and white CCD camera using Axiovision software (Carl Zeiss, Oberkochen, Germany). Live images for Fig. S3 were acquired on an inverted Leica DMi8 epifluorescence microscope equipped with a 20x phase-contrast objective and a Leica K5 camera using Thunder Imaging System. All images were uniformly processed for brightness and contrast using Adobe Photoshop (San Jose, USA).

\section{Immunohistochemistry on squashed testis preparations} immunofluorescence as previously described (Hime et al., 1996). In brief, after mounting on polylysine coated slides (Polysine, Thermo Fisher Scientific, P4681-001, Waltham, USA), samples were squashed with a coverslip and frozen in liquid nitrogen. Coverslips were removed

511 Samples were fixed in PBS with 4\% paraformaldehyde (Electron Microscope Sciences, 15710,

512 Hadfield, USA) for 7-10 minutes, permeabilized in PBS with 0.37\% Triton X-100 and 0.3\%

513 sodium deoxycholate for 30 minutes, and blocked in PBS with 0.1\% Triton X-100 and 5\%

514 bovine serum albumin (PBTB; Sigma-Aldrich, A3912-100G, St. Louis, USA). Samples were 515 incubated at $4{ }^{\circ} \mathrm{C}$ overnight with primary antibodies, then washed with PBTB three times for 5

516 minutes and once for 15 minutes and incubated for 1 hour at room temperature with secondary 517 antibodies diluted in PBTB. Samples were then washed with PBTB once for 15 minutes, stained 518 (when applicable) with rhodamine-phalloidin in PBTB (1:200; Invitrogen, R415) for 30 minutes, 519 washed with PBS with 0.1\% Triton X-100 (PBT) for 15 minutes, stained with 4',6-diamidino-2520 phenylindole (DAPI) in PBT (1:1000; VWR, 89139-054, Radnor, USA) for 10 minutes and 521 washed with PBT twice for 15 minutes. Samples were mounted in ProLong Diamond Antifade 522 Mountant (Molecular Probes, P36961, Eugene, USA), sealed with nail polish, and examined 523 within 1-2 days. Fluorescence micrographs were acquired on a Nikon A1R inverted laser 
524 scanning confocal equipped with 10x, 20x, 40x, and 60x objectives, photomultiplier tube (PMT)

525 detectors for DAPI channel, and gallium arsenide phosphide (GaAsP) PMT detectors for green

526 and red channels using NIS Elements software (SickKids Imaging Facility, The Hospital for Sick

527 Children, Toronto, Canada). All images were uniformly processed for brightness and contrast

528 using Adobe Photoshop (San Jose, USA). Apart from Fig. 3H, all images from the same

529 experiment were adjusted for brightness and contrast in an identical manner.

$530 \quad$ Primary antibodies used for immunofluorescence were rabbit anti-eIF4E-5 (1:500; \#4524,

531 this work), rabbit anti-caspase-3 (1:400; Asp175, Cell Signaling Technology, Danvers, USA),

532 guinea pig anti-Soti (1:100; Kaplan et al., 2010; a kind gift of Eli Arama, Weitzmann Institute,

533 Rohovot, Israel), mouse anti-Adducin 1B1 (1:20; Zaccai and Lipshitz, 1996; a kind gift of

534 Howard Lipshitz, University of Toronto, Toronto, Canada) and mouse anti-FLAG (1:200; M2,

535 Sigma-Aldrich, F1804). Secondary antibodies used for immunofluorescence were Alexa Fluor

536 488-conjugated anti-rabbit (1:1000; Invitrogen, A-11008), Alexa Fluor 488-conjugated anti-

537 guinea pig (1:1000; Invitrogen, A-11073), or Alexa Fluor 568-conjugated anti-mouse IgG

538 (1:1000; Invitrogen, A-31043).

539

\section{Quantifying spermatid cyst polarity defects}

542 described above. The percentage of testes with spermatid nuclei near the tip was recorded (Xu et

543 al., 2014). For Fig. 7E-F, statistical analysis and graphing were performed using GraphPad Prism

544 versions 8 for Macintosh, respectively (GraphPad Software). Differences observed between

545 genotypes were analyzed with unpaired two-tailed Student $t$-test. Results were considered

546 statistically significant at $p<0.05$.

\section{Immunofluorescence with single molecule RNA FISH}

All solutions used were RNase free. Testes were dissected in 1xPBS (Invitrogen,

550 AM9624) and fixed in 4\% formaldehyde (Polysciences, Inc., 18814-10) in 1xPBS for 30

551 minutes, washed briefly in PBS, and permeabilized in $70 \%$ ethanol overnight at $4^{\circ} \mathrm{C}$. Samples

552 were then washed with 1 xPBS and blocked for 30 minutes at $37^{\circ} \mathrm{C}$ in blocking buffer $(1 \mathrm{xPBS}$,

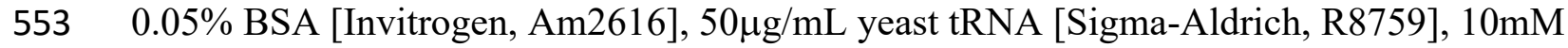

554 Vanadyl Ribonucleoside complex [New England Biolabs, S1402S], 0.2\% Tween-20 [Sigma- 
555

556

557

558

559

560

561

562

563

564

565

566

567

568

569

570

571

572

573

574

575

576

577

578

579

580

581

582

583

584

585

Aldrich, P7949]). Samples were incubated with primary antibodies diluted in blocking buffer overnight at $4{ }^{\circ} \mathrm{C}$, washed with $1 \mathrm{xPBS}$ containing $0.2 \%$ Tween-20, re-blocked for 5 minutes at $37^{\circ} \mathrm{C}$ in blocking buffer, and incubated $4^{\circ} \mathrm{C}$ overnight in blocking buffer containing secondary antibodies. Testes were then washed with $1 \times$ PBS containing $0.2 \%$ Tween-20, and re-fixed for 10 minutes before being briefly rinsed with wash buffer (2x saline-sodium citrate [SSC, Invitrogen, AM9770], 10\% formamide [Fisher Scientific, BP227]), and then hybridized overnight at $37^{\circ} \mathrm{C}$ in hybridization buffer (2xSSC, 10\% dextran sulfate [Sigma-Aldrich, D8906], 1mg/mL yeast tRNA, 2mM Vanadyl Ribonucleoside complex, 0.5\% BSA, 10\% formamide). Following hybridization, samples were washed three times in wash buffer for 20 minutes each at $37^{\circ} \mathrm{C}$ and mounted in Vectashield with DAPI (Vector Laboratory, H-1200, Burlingame, USA). Images were acquired using an upright Leica Stellaris 8 confocal microscope with a $63 \mathrm{X}$ oil immersion objective lens $(\mathrm{NA}=1.4)$ and processed using ImageJ software (National Institutes of Health, Bethesda, USA).

Primary antibodies were rabbit anti-eIF4E-5 (1:500; \#4524, this work) or rabbit antiFLAG (1:500; Invitrogen, PA1-984B), and secondary antibodies were Alexa Fluor 488conjugated anti-rabbit (1:200; Life Technologies, Carlsbad, USA). Fluorescently labeled probes were added to the hybridization buffer to a final concentration of $100 \mathrm{nM}$. Probes against $k l-3$, soti, and eIF4E-5 mRNA were designed using the Stellaris ${ }^{\circledR}$ RNA FISH Probe Designer (Biosearch Technologies, Inc., Novato, USA) available online at www.biosearchtech.com/stellarisdesigner. Probe sets are listed in Table S1.

For smFISH alone, hybridization immediately followed the overnight incubation in $70 \%$ ethanol and short wash with wash buffer.

\section{Immunoblotting}

Three methods were used for immunoblotting. For Fig. 1, approximately 20 pairs of testes per genotype were dissected in TIB with protease inhibitor cocktail (1:100; Halt, Thermo Fisher Scientific, 87786), then lysed in radioimmunoprecipitation assay (RIPA) buffer with protease inhibitors $(150 \mathrm{mM} \mathrm{NaCl}, 50 \mathrm{mM}$ Tris- $\mathrm{HCl} \mathrm{pH} 8.0,1 \%$ Nonidet P-40, 0.5\% sodium deoxycholate, $0.1 \%$ SDS). NuPAGE LDS Sample Buffer was added, and samples were boiled for 10 minutes at $98^{\circ} \mathrm{C}$ (Invitrogen, NP0007). Proteins were run on gradient pre-cast SDS polyacrylamide gels (8-16\%, ExpressPlu, GenScript, M81610, Piscataway, USA) before being 
586

587

588

589

590

591

592

593

594

595

596

597

598

599

600

601

602

603

604

605

606

607

608

609

610

611

612

613

614

615

616

transferred to nitrocellulose membranes $(0.45 \mu \mathrm{m}$, Amersham Protran, GE Healthcare Life Sciences, 10600020 Chicago, USA) with NuPAGE Transfer Buffer (Invitrogen, NP0006). Membranes were rinsed in TBST (Tris-buffered saline with 0.05\% Tween-20), blocked in TBST containing $5 \%$ nonfat milk, and incubated overnight at $4{ }^{\circ} \mathrm{C}$ with primary antibodies diluted in TBST containing 5\% nonfat milk. Membranes were washed with TBST and incubated with secondary antibodies diluted in TBST containing 1\% nonfat milk. Membranes were washed with TBST, and detection was performed using Novex ECL Chemiluminescent Substrate Reagents Kit (Invitrogen, WP20005). Primary antibodies used were rabbit anti-eIF4E-5 (1:5000; \#4524, this work) and mouse anti- $\alpha$-tubulin ( $5 \mathrm{ug} / \mathrm{mL}$; AA4.3, Developmental Studies Hybridoma Bank, Iowa City, USA). Secondary antibodies were HRP-conjugated anti-rabbit (1:10,000; Jackson ImmunoResearch Laboratories, 111-035-003, West Grove, USA) or anti-mouse IgG (1:10,000; Jackson ImmunoResearch Laboratories, 715-035-150).

For Fig. 6, testes (40 pairs/sample) were dissected in Schneider's medium (Gibco, 21720 024) at room temperature within 30 minutes, the medium was removed, and samples were frozen at $-80^{\circ} \mathrm{C}$ until use. Tissues were then lysed in equal volumes of $2 x L a e m m l i$ Sample Buffer (BioRad Laboratories, 1610737, Hercules, USA) + $\beta$ ME (Bio-Rad Laboratories, 1610710, Hercules, USA) and equal volumes were run on a NuPAGE Tris-Acetate gel (3-8\%, 1.5mm, Invitrogen, EA0378BOX) with Tris-Acetate SDS Running Buffer (Invitrogen, LA0041) before being transferred onto polyvinylidene fluoride (PVDF) membrane (Bio-Rad Laboratories, 1620177, Hercules, USA) using NuPAGE transfer buffer (Invitrogen, NP0006) without added methanol. Membranes were blocked in 1xTBST (0.1\% Tween-20) containing 5\% nonfat milk (Bio-Rad, 1706404, Hercules, USA), followed by incubation with primary antibodies diluted in 1X TBST containing 5\% nonfat milk. Membranes were then washed with 1xTBST, followed by incubation with secondary antibodies diluted in 1xTBST containing 5\% nonfat milk. After washing with 1xTBST, detection was performed using the Pierce ${ }^{\circledR}$ ECL Western Blotting Substrate enhanced chemiluminescence system (Thermo Fisher Scientific, 32106). Primary antibodies used were mouse anti- $\alpha$-tubulin (1:2,000; clone DM1a, Sigma-Aldrich) and mouse anti-FLAG (1:2,500; M2, Sigma-Aldrich). Secondary antibody was HRP-conjugated anti-mouse IgG (1:10,000; Abcam, ab6789, Cambridge, UK).

For Fig. S6, immunoblotting was carried out as described for Fig. 6 except that samples were run on a Novex Tris-Glycine gel (14\%, 1mm, Invitrogen, XP00140BOX) with running 
617 buffer (25mM Tris base, $192 \mathrm{mM}$ glycine, 0.1\% SDS) and transferred to the PVDF membrane

618 using transfer buffer (25mM Tris base, 192mM glycine, 20\% methanol). Primary antibodies used

619 were mouse anti- $\alpha$-tubulin (1:2,000; clone DM1a, Sigma-Aldrich) and rabbit anti-eIF4E-5

620 (1:5000; \#4524, this work). Secondary antibodies were HRP-conjugated anti-mouse IgG

621 (1:10,000; Abcam, ab6789, Cambridge, UK) and HRP-conjugated anti-rabbit IgG (1:10,000;

622 Abcam, ab6721, Cambridge, UK).

Yeast two-hybrid system

A cDNA encoding Drosophila eIF4E-5 (CG8277) was PCR-amplified using the plasmid 4E5-pCR2.1 as a template (Hernández et al., 2005) and subcloned into the vector pOAD ("prey"

627 vector; Cagney et al., 2000) in-frame with the activator domain sequence of GAL4 to generate

628 the construct eIF4E-5-AD. Drosophila GRB10-interacting GYF (glycine-tyrosine-phenylalanine 629 domain) protein (GIGYF, CG11148; Russica et al., 2019; a kind gift of Catia Igreja, Max Planck 630 for Developmental Biology, Tübingen, Germany), CUP (CG11181; Nelson et al., 2004;

631 Zappavigna, et al., 2004; a kind gift of Nancy Standart, Cambridge University, Cambridge, UK), 632 eIF4E transporter (4E-T, CG32016), eIF4G (CG10811; Hernández et al. 1998), eIF4G-2

633 (CG10192; Baker and Fuller 2007), and 4E-BP (CG8846; Miron et al., 2001) cDNAs were 634 subcloned into the pOBD2 vector ("bait" vector; Cagney et al., 2000) in-frame with the DNA635 binding domain sequence of GAL4 to create the respective plasmids pGIGYF-BD, pCUP-BD, 636 p4E-T-BD, peIF4G-2 (313-1164)-BD, peIF4G-BD and p4E-BP-BD.

637 Interactions between proteins expressed as "prey" or "bait" fusions were detected 638 following a yeast interaction-mating method using the strains PJ69-4a and PJ69-4 $\alpha$ (Cagney et 639 al., 2000). Diploid cells containing both bait and pray plasmids were grown in -Trp, -Leu 640 selective media (Clontech, 630417, Mountain View, USA) and shown as controls for growth.

641 Protein interactions were detected by replica-plating diploid cells onto -Trp, -Leu, -Ade (20

$642 \mathrm{ug} / \mathrm{mL}$ L-His HCl monohydrate (A-9126, Sigma) added to -Trp, -Leu, -Ade, -His (630428, 643 Clontech) or -Trp, -Leu, -His (630419, Clontech) selective media +3 mM, 10 mM or 30 mM 3644 amino-1,2,4-triazole (3AT, Sigma-Aldrich). Growth was scored after 4 days of growth at $30^{\circ} \mathrm{C}$. 


\section{Acknowledgments}

647 The authors are grateful to Brill lab members Jonathan Ma, Nigel Giffiths, Lacramioara Fabian,

648 Alind Gupta and Yonit Bernstein for their support and assistance with experimental methods. We

649 thank Eli Arama and Howard Lipshitz for antibodies. We also thank Howard Lipshitz, Craig

650 Smibert and James Ellis for helpful discussions, Helen White-Cooper for insightful comments on

651 the manuscript, Paul Paroutis and Kimberly Lau of the SickKids Imaging facility for advice on

652 imaging, and the Bloomington Drosophila Stock Center for fly stocks.

653

654 Conflict of interest

655 The authors declare no competing interests.

656

657 Author contributions

658 Conceptualization, L.S., J.A.B.; Methodology, L.S., J.M.F., B.L.F., H.H., G.M., Y.Q., V.L.,

659 E.H., L.C., G.P., G.H., P.L., J.A.B.; Validation, L.S., J.M.F., B.L.F., G.M., G.H., J.A.B.; Formal

660 analysis, L.S., J.M.F., B.L.F., J.A.B.; Investigation, L.S., J.M.F., B.L.F., G.M., V.L., E.H., L.C.,

661 J.A.B.; Resources, H.H., P.L.; Writing - original draft, L.S., J.A.B.; Writing - review and

662 editing, all authors; Visualization, L.S., J.M.F., B.L.F., G.M., G.H., J.A.B.; Supervision, L.S.,

663 G.P., G.H., P.L., J.A.B; Project administration, L.S., J.A.B.; Funding acquisition, G.H., P.L.,

664 J.A.B.

665

666 Funding

667 This work was supported by SickKids Restracomp and Ontario Graduate Scholarships (to L.S.);

668 National Council of Science and Technology (CONACyT) Ph.D. fellowship \#749487 (to G.M.);

669 intramural funding program of the Instituto Nacional de Cancerología, Mexico (to G.H.); CIHR

670 Research Grant \#IOP-107945 (to P.L.); and NSERC Discovery (\#RGPIN-2016-06775) and

671 Research Tools and Instruments (\#RTI-2019-00361) grants (to J.A.B.). 


\section{References}

674 Amiri, A., Keiper, B.D., Kawasaki, I., Fan, Y., Kohara, Y., Rhoads, R.E., and Strome, S. (2001). An isoform of eIF4E is a component of germ granules and is required for spermatogenesis in C. elegans. Development 128, 3899-3912.

Aguilar-Valles, A., Haji, N., De Gregorio, D., Matta-Camacho, E., Eslamizade, M.J., Popic, J., depression-like behavior via phosphorylation of eukaryotic translation initiation factor 4E. Nat Commun 25, 2459.

Arama, E., Agapite, J., and Steller, H. (2003). Caspase activity and a specific cytochrome C are required for sperm differentiation in Drosophila. Dev Cell 4, 687-697.

Arama, E., Bader, M., Rieckhof, G.E., and Steller, H. (2007). A ubiquitin ligase complex regulates caspase activation during sperm differentiation in Drosophila. PLoS Biol 5, e251.

Ashburner, M. (1990). Drosophila: A laboratory handbook (Cold Spring Harbor, NY: Cold Spring Harbor Press).

Baker, C.C., and Fuller, M.T. (2007). Translational control of meiotic cell cycle progression and spermatid differentiation in male germ cells by a novel eIF4G homolog. Development 134, 2863-2869.

Barr, J., Yakovlev, K.V., Shidlovskii, Y., and Schedl, P. (2016). Establishing and maintaining cell polarity with mRNA localization in Drosophila. BioEssays 38, 244-253.

Barreau, C., Benson, E., Gudmannsdottir, E., Newton, F., and White-Cooper, H. (2008a). Postmeiotic transcription in Drosophila testes. Development 135, 1897-1902.

Barreau, C., Benson, E., White-Cooper, H. (2008b). Comet and cup genes in Drosophila spermatogenesis: the first demonstration of post-meiotic transcription. Biochem Soc Trans $36,540-542$. interactions using two-hybrid assay. Methods Enzymol 328, 3-14. 
Casal, J., Gonzalez, C., Wandosell, F., Avila, J., and Ripoll, P. (1990). Abnormal meiotic spindles cause a cascade of defects during spermatogenesis in asp males of Drosophila. Development 108, 251-260.

Chintapalli, V.R., Wang, J., and Dow, J.A.T. (2007). Using FlyAtlas to identify better Drosophila melanogaster models of human disease. Nat Genet 39, 715-720.

Dinkova, T.D., Keiper, B.D., Korneeva, N.L., Aamodt, E.J., and Rhoads, R.E. (2005). Translation of a small subset of Caenorhabditis elegans mRNAs is dependent on a specific eukaryotic translation initiation factor 4E isoform. Mol Cell Biol 25, 100-113.

Fabian, L., and Brill, J.A. (2012). Drosophila spermiogenesis: Big things come from little packages. Spermatogenesis 2, 197-212.

Fingerhut, J.M., Moran, J.V., Yamashita, Y.M. (2019). Satellite DNA-containing gigantic introns in a unique gene expression program during Drosophila spermatogenesis. PLoS Genet 15, e1008028.

Fingerhut, J.M., Yamashita, Y.M. (2020). mRNA localization mediates maturation of cytoplasmic cilia in Drosophila spermatogenesis. J Cell Biol 219, e202003084.

Franklin-Dumont, T.M., Chatterjee, C., Wasserman, S.A., DiNardo, S. (2007). A novel eIF4G homolog, off-schedule, couples translational control to meiosis and differentiation in Drosophila spermatocytes. Development 134, 2851-2861.

Fuller, M.T. (1993). Spermatogenesis (Cold Spring Harbor, NY: Cold Spring Harbor Press).

Ge, D.T., Tipping, C., Brodsky, M.H., and Zamore, P.D. (2016). Rapid Screening for CRISPRDirected Editing of the Drosophila Genome Using white Co-conversion. G3 (Bethesda) 6, 3197-3206.

Ghosh, S., and Lasko, P. (2015). Loss-of-function analysis reveals distinct requirements of the translation initiation factors eIF4E, eIF4E-3, eIF4G and eIF4G2 in Drosophila spermatogenesis. PLoS ONE 10, e0122519.

Gottardo, M., Callaini, G., and Riparbelli, M.G. (2013). The cilium-like region of the Drosophila spermatocyte: an emerging flagellum? J Cell Sci 126, 5441-5452.

Gratz, S.J., Ukken, F.P., Rubinstein, C.D., Thiede, G., Donohue, L.K., Cummings, A.M., and O'Connor-Giles, K.M. (2014). Highly Specific and Efficient CRISPR/Cas9-Catalyzed Homology-Directed Repair in Drosophila. Genetics 196, 961-971. 
732

733

734

735

736

737

738

739

740

741

742

743

744

745

746

747

748

749

750

751

752

753

754

755

756

757

758

759

760

761

762

Graveley, B.R., Brooks, A.N., Carlson, J.W., Duff, M.O., Landolin, J.M., Yang, L., Artieri, C.G., van Baren, M.J., Boley, N., Booth, B.W., et al. (2011). The developmental transcriptome of Drosophila melanogaster. Nature 471, 473-479.

Grüner, S., Peter, D., Weber, R., Wohlbold, L., Chung, M., Weichenrieder, O., Valkov, E., Igreja, C., and Izaurralde, E. (2016). The Structures of eIF4E-eIF4G Complexes Reveal an Extended Interface to Regulate Translation Initiation. Mol Cell 64, 467-479.

Henderson, M.A., Cronland, E., Dunkelbarger, S., Contreras, V., Strome, S., and Keiper, B.D. (2009). A germline-specific isoform of eIF4E (IFE-1) is required for efficient translation of stored mRNAs and maturation of both oocytes and sperm. J Cell Sci 122, 1529-1539.

Hengst, U., Deglincerti, A., Kim, H.J., Jeon, N.L., and Jaffrey, S.R. (2009). Axonal elongation triggered by stimulus-induced local translation of a polarity complex protein. Nat Cell Biol 11, 1024-1030.

Hernández, G., Castellano, M.M., Agudo, M., Sierra, J.M. (1998). Isolation and characterization of the cDNA and the gene for eukaryotic translation initiation factor $4 \mathrm{G}$ from Drosophila melanogaster. Eur J Biochem 253, 27-35.

Hernández, G., Altmann, M., Sierra, J.M., Urlaub, H., Diez del Corral, R., Schwartz, P., and Rivera-Pomar, R. (2005). Functional analysis of seven genes encoding eight translation initiation factor 4E (eIF4E) isoforms in Drosophila. Mech Dev 122, 529-543.

Hernández, G., Han, H., Gandin, V., Fabian, L., Ferreira, T., Zuberek, J., Sonenberg, N., Brill, J.A., and Lasko, P. (2012). Eukaryotic initiation factor 4E-3 is essential for meiotic chromosome segregation, cytokinesis and male fertility in Drosophila. Development 139, 3211-3220.

Hershey, J.W.B., Sonenberg, N., and Mathews, M.B. (2019). Principles of Translational Control. Cold Spring Harb Perspect in Biol 11, a032607.

Hime, G.R., Brill, J.A., and Fuller, M.T. (1996). Assembly of ring canals in the male germ line from structural components of the contractile ring. J Cell Sci 109, 2779-2788.

Huh, J.R., Vernooy, S.Y., Yu, H., Yan, N., Shi, Y., Guo, M., and Hay, B.A. (2004). Multiple apoptotic caspase cascades are required in nonapoptotic roles for Drosophila spermatid individualization. PLoS Biol 2, E15.

Jayaramaiah-Raja, S., and Renkawitz-Pohl, R. (2005). Replacement by Drosophila melanogaster protamines and Mst77F of histones during chromatin condensation in late spermatids and 
role of sesame in the removal of these proteins from the male pronucleus. Mol Cell Biol 25, 6165-6177.

Joshi, B., Cameron, A., Jagus, R. (2004). Characterization of mammalian eIF4E-family members. Eur J Biochem 271, 2189-203.

Kamenska, A., Lu, W.T., Kubacka, D., Broomhead, H., Minshall, N., Bushell, M., Standart, N. (2014). Human 4E-T represses translation of bound mRNAs and enhances microRNAmediated silencing. Nucleic Acids Res 42, 3298-3313.

Kaplan, Y., Gibbs-Bar, L., Kalifa, Y., Feinstein-Rotkopf, Y., and Arama, E. (2010). Gradients of a ubiquitin E3 ligase inhibitor and a caspase inhibitor determine differentiation or death in spermatids. Dev Cell 19, 160-173.

Kawasaki, I., Jeong, M., and Shim, Y. (2011). Regulation of sperm-specific proteins by IFE-1, a germline-specific homolog of eIF4E, in C. elegans. Mol Cells 31, 191-197.

Keiper, B.D., Lamphear, B.J., Deshpande, A.M., Jankowska-Anyszka, M., Aamodt, E.J., Blumenthal, T., and Rhoads, R.E. (2000). Functional characterization of five eIF4E isoforms in Caenorhabditis elegans. J Biol Chem 275, 10590-10596.

Kugler, J., and Lasko, P. (2009). Localization, anchoring and translational control of oskar, gurken, bicoid and nanos mRNA during Drosophila oogenesis. Fly (Austin) 3, 15-28.

Lin, A.C., and Holt, C.E. (2007). Local translation and directional steering in axons. Embo J 26, 3729-3736.

Lindsley, D.T., and Tokuyasu, K.T. (1980). Normal spermatogenesis in Drosophila (New York: Academic Press).

Macara, I.G., Iioka, H., and Mili, S. (2009). Axon growth-stimulus package includes local translation. Nat Cell Biol 11, 919-921.

Mingle, L.A., Okuhama, N.N., Shi, J., Singer, R.H., Condeelis, J., and Liu, G. (2005). Localization of all seven messenger RNAs for the actin-polymerization nucleator Arp2/3 complex in the protrusions of fibroblasts. J Cell Sci 118, 2425-2433.

Miron, M., Verdú, J., Lachance, P.E., Birnbaum, M.J., Lasko, P.F., Sonenberg, N. (2001). The translational inhibitor 4E-BP is an effector of PI(3)K/Akt signalling and cell growth in Drosophila. Nat Cell Biol 3, 596-601.

Moor, A.E., Golan, M., Massasa, E.E., Lemze, D., Weizman, T., Shenhav, R., Baydatch, S., Mizrahi, O., Winkler, R., Golani, O., Stern-Ginossar, N., and Itzkovitz, S. (2017). Global 
mRNA polarization regulates translation efficiency in the intestinal epithelium. Science 357, 1299-1303.

Muro, I., Berry, D.L., Huh, J.R., Chen, C.H., Huang, H., Yoo, S.J., Guo, M., Baehrecke, E.H., and Hay, B.A. (2006). The Drosophila caspase Ice is important for many apoptotic cell deaths and for spermatid individualization, a nonapoptotic process. Development 133 , 3305-3315.

Nelson, M.R., Leidal, A.M., and Smibert, C.A. (2004). Drosophila Cup is an eIF4E-binding protein that functions in Smaug-mediated translational repression. Embo J 23, 150-159.

Pelechano, V., Wei, W., and Steinmetz, L.M. (2015). Widespread co-translational RNA decay reveals ribosome dynamics. Cell 161, 1400-1412.

Pelletier, J., and Sonenberg, N. (2019). The organizing principles of eukaryotic ribosome recruitment. Annu Rev Biochem 88, 307-335.

Perkins, L.A., Holderbaum, L., Tao, R., Hu, Y., Sopko, R., McCall, K., Yang-Zhou, D., Flockhart, I., Binari, R., Shim, H.S., et al. (2015). The transgenic RNAi project at Harvard Medical School: Resources and validation. Genetics 201, 843-852.

Rengan, A.K., Agarwal, A., van der Linde, M., and du Plessis, S.S. (2012). An investigation of excess residual cytoplasm in human spermatozoa and its distinction from the cytoplasmic droplet. Reprod Biol Endocrinol 10:92.

Renkawitz-Pohl, R., Hempel, L., Hollmann, M., Schäfer, M.A. (2005). Spermatogenesis. In Comprehensive Insect Physiology, Biochemistry, Pharmacology and Molecular Biology (ed. L.I. Gilbert, K. Iatrou, S. Gill), pp. 157-78. Oxford: Elsevier. cilia in dividing Drosophila spermatocytes. Dev Cell 23, 425-432. 
823 Santel, A., Winhauer, T., Blümer, N., Renkawitz-Pohl, R. (1997). The Drosophila don juan (dj)

824 gene encodes a novel sperm specific protein component characterized by an unusual domain of a repetitive amino acid motif. Mech Dev 64, 19-30.

Schäfer, M., Börsch, D., Hülster, A., and Schäfer, U. (1993). Expression of a gene duplication encoding conserved sperm tail protein is translationally regulated in Drosophila melanogaster. Mol Cell Biol 13, 1708-1718.

Shaha, C., Tripathi, R., and Mishra, D.P. (2010). Male germ cell apoptosis: regulation and biology. Philos Trans R Soc Lond B Biol Sci 365(1546):1501-1515.

Sonenberg, N., and Hinnebusch, A.G. (2009). Regulation of Translation Initiation in Eukaryotes: Mechanisms and Biological Targets. Cell 136, 731-745.

Song, A., Labella, S., Korneeva, N.L., Keiper, B.D., Aamodt, E.J., Zetka, M., and Rhoads, R.E. (2010). A C. elegans eIF4E-family member upregulates translation at elevated temperatures of mRNAs encoding MSH-5 and other meiotic crossover proteins. $J$ Cell Sci $123,2228-2237$.

Tokuyasu, K.T. (1975). Dynamics of spermiogenesis in Drosophila melanogaster. VI. Significance of "onion" nebenkern formation. J Ultrastruct Res 53, 93-112.

Tuck, A.C., Rankova, A., Arpat, A.B., Liechti, L.A., Hess, D., Lesmantavicius, V., CasteloSzekely, V., Gatfield, D., and Buhler, M. (2020). Mammalian RNA decay pathways are highly specialized and widely linked to translation. Mol Cell 77, 1222-1236.

Vibranovski, M.D., Chalopin, D.S., Lopes, H.F., Long, M., and Karr, T.L. (2010). Direct evidence for postmeiotic transcription during Drosophila melanogaster spermatogenesis. Genetics 186, 431-433.

Vibranovski, M.D., Lopes, H.F., Karr, T.L., and Long, M. (2009). Stage-specific expression profiling of Drosophila spermatogenesis suggests that meiotic sex chromosome inactivation drives genomic relocation of testis-expressed genes. PLoS Genet 5, e1000731.

Wiebe, S., Meng, X.Q., Kim, S.H., Zhang, X., Lacaille, J.C., Aguilar-Valles, A., Sonenberg, N. (2020). The eIF4E homolog 4EHP (eIF4E2) regulates hippocampal long-term depression and impacts social behavior. Mol Autism 11, 92.

White-Cooper, H. (2010). Molecular mechanisms of gene regulation during Drosophila spermatogenesis. Reproduction 139, 11-21. 
853 White-Cooper, H., and Caporilli, S. (2013). Transcription and post-transcriptional regulation of

854 Drosophila germline stem cells and their differentiating progeny. In Transcriptional and

855 Translational Regulation of Stem Cells (ed. G. Hime and H. Abud), pp. 47-61. Dordrecht:

856 Springer.

857 Xu, S., Hafer, N., Agunwamba, B., and Schedl, P. (2012). The CPEB protein Orb2 has multiple 858 functions during spermatogenesis in Drosophila melanogaster. PLoS Genet 8, e1003079.

859 Xu, S., Tyagi, S., and Schedl, P. (2014). Spermatid cyst polarization in Drosophila depends upon 860 apkc and the CPEB family translational regulator orb2. PLoS Genet 10, e1004380.

861 Zaccai, M., Lipshitz, H.D. (1996). Role of Adducin-like (hu-li tai shao) mRNA and protein 862 localization in regulating cytoskeletal structure and function during Drosophila oogenesis 863 and early embryogenesis. Dev Genet 19, 249-257.

864 Zappavigna, V., Piccioni, F., Villaescusa, J.C., Verrotti, A.C. (2004). Cup is a nucleocytoplamic 865 shuttling protein that interacts with the eukaryotic translation initiation factor 4E to modulate Drosophila ovary development. Proc Natl Acad Sci U S A 101, 14800-14805.

867 Zhao, J., Klyne, G., Benson, E., Gudmannsdottir, E., White-Cooper, H., Shotton, D. (2010). FlyTed: the Drosophila testis gene expression database. Nucleic Acids Res 38, D710-5.

869 Zhou, X., Fabian, L., Bayraktar, J.L., Ding, H-M., Brill, J.A., Chang, H.C. (2011). Auxilin is required for formation of Golgi-derived clathrin-coated vesicles during Drosophila

872 Zuberek, J., Kuchta, K., Hernández, G., Sonenberg, N., Ginalski, K. (2016). Diverse cap-binding properties of Drosophila eIF4E isoforms. Biochim Biophys Acta 1864, 1292-1303. 


\section{Figure Legends}

877 Fig. 1. eIF4E-5 mutants are male sterile. (A) CRISPR/Cas9 mutagenesis was used to generate 878 mutants of eIF4E-5. Diagram showing nucleotide position of eIF4E-5 on chromosome 3L (top).

879 Gene structure: 5' and 3' UTRs (grey boxes), coding exons (black boxes), introns (lines). Locations of gRNAs in 5'UTR (gRNA1) and first exon (gRNA2) are indicated. eIF4E-5 ${ }^{B 8 a}$, $e I F 4 E-5^{B 8 b}$ and $e I F 4 E-5^{D 19 a}$ mutants contain deletions in the first exon, overlapping gRNA2.

882 Genomic sequence: protospacer adjacent motif (blue), target region of the gRNA (red), deletions (dashed lines). (B) Diagram showing structure of predicted wild-type (top) and mutant eIF4E-5 proteins. Wild-type eIF4E-5 has a non-conserved N-terminus (amino acids 1-54; white box) and conserved C-terminus (amino acids 54-232; dark grey box). In-frame deletions encoded by $e I F 4 E-5^{B 8 a}$ and $e I F 4 E-5^{B 8 b}$ and frameshift (fs) mutation encoded by eIF4E-5 $5^{D 19 a}$ (out-of-frame amino acids 56-77; light grey box) are indicated. (C) Immunoblot of total testis extracts probed with the indicated antibodies reveal reduced $\left(e I F 4 E-5^{B 8 a}\right.$ and $\left.e I F 4 E-5^{B 8 b}\right)$ or undetectable $\left(e I F 4 E-5^{D 19}\right)$ levels of eIF4E-5 protein. Note that these levels correlate with the severity of the phenotypes shown in Figs 2, 3 and 7. (D) Fertility tests reveal that all tested combinations of eIF4E-5 alleles and deficiencies are male sterile and that a genomic transgene expressing 3xFLAG-eIF4E-5 restores fertility to $e I F 4 E-5^{B 8 b}$ and $e I F 4 E-5^{D 19 a}$ mutant males $\left(e I F 4 E-5^{B 8 a}\right.$ not tested). (E-H) Laser-scanning confocal fluorescence micrographs demonstrate the presence of needle-shaped sperm nuclei in wild-type (E) but not $e I F 4 E-5^{B 8 a}(\mathrm{~F}), e I F 4 E-5^{B 8 b}(\mathrm{G})$ or $e I F 4 E-$ $5^{D 19 a}(\mathrm{H})$ seminal vesicles stained with DAPI. Scale bars: $20 \mu \mathrm{m}$.

Fig. 2. eIF4E-5 localizes to the distal ends of elongated spermatid cysts. Laser-scanning confocal fluorescence micrographs (A-D) Wild-type (A), eIF4E-5 $5^{B 8 a}(\mathrm{~B}), e I F 4 E-5^{B 8 b}(\mathrm{C})$ and $e I F 4 E-5^{D 19 a}$ (D) whole adult testes stained for Adducin (magenta, red arrowheads) and eIF4E-5 (green, yellow arrowheads) reveals that eIF4E-5 localizes just distal to Adducin at the ends of 901 elongated spermatid cysts in wild-type but not eIF4E-5 mutant spermatid cysts. Note that antieIF4E-5 antibodies non-specifically stain individualization complexes, as shown for the mispolarized cyst in eIF4E-5 ${ }^{B 8 b}$ (C-C', , cyan arrowheads). Scale bars: $20 \mu \mathrm{m}$. (E-H) $3 x-F L A G-$ $904 e I F 4 E-5 ; e I F 4 E-5^{D 19 a}(\mathrm{E}, \mathrm{G})$ and $e I F 4 E-5^{D 19 a}(\mathrm{~F}, \mathrm{H})$ whole adult testes stained for 3xFLAG- 
at the distal end of elongated spermatid cysts (E; orange arrowheads). Note non-specific staining of spermatogonia and spermatid tails with anti-FLAG antibodies and non-specific staining of nuclei in spermatogonia, spermatocytes and round spermatids with anti-eIF4E-5 antibodies (E$\left.\mathrm{H}^{\prime \prime}\right)$ in eIF4E-5 $5^{D 19 a}$ mutants. Scale bars: $20 \mu \mathrm{m}(\mathrm{E}-\mathrm{F}), 100 \mu \mathrm{m}(\mathrm{G}-\mathrm{H})$.

Fig. 3. eIF4E-5 mutants exhibit defects in individualization. (A-D) Phase-contrast images of 4-day old wild-type (A), eIF4E-5 $5^{B 8 a}$ (B), eIF4E-5 $5^{B 8 b}$ (C) or eIF4E-5 $5^{D 19 a}$ (D) testes reveal an absence of waste bags (red arrowheads) near the testis tip in eIF4E-5 mutants. Scale bars: 100 $\mu \mathrm{m}$. (E-H) Laser-scanning confocal fluorescence micrographs of wild type (E), eIF4E-5 ${ }^{B 8 a}(\mathrm{~F})$, $\operatorname{eIF} 4 E-5^{B 8 b}(\mathrm{G})$ or $e I F 4 E-5^{D 19 a}(\mathrm{H})$ whole adult testes stained for DNA (magenta), activated caspase-3 (green) and F-actin (shown only in insets). Activated caspase (yellow arrowheads) is restricted to cystic bulges in wild type (E) but not eIF4E-5 mutants (F-H). Boxed areas are magnified 10-fold in insets. Groups of actin cones in individualization complexes move synchronously down the length of cysts in wild type (E',') but become scattered prior to reaching the distal end of elongated spermatid cysts in eIF4E-5 mutants (F', '-H', '). Brightness and contrast for Fig. 3H', were adjusted separately from the rest of the images for visualizing individualization complexes. Scale bars: $200 \mu \mathrm{m}$ (whole tissue), $20 \mu \mathrm{m}$ (insets). scanning confocal fluorescence micrographs. (A-C) Wild-type (A), eIF4E-5 $5^{D 19 a}(\mathrm{~B})$ and $3 x F L A G-e I F 4 E-5 ; e I F 4 E-5^{D 19 a}$ rescue (C) whole adult testes stained for DNA (magenta) and Soti elongated spermatid cysts in wild type and rescue but not eIF4E-5 $5^{D 19 a}$ (yellow arrowheads). Note presence of nuclei from mispolarized spermatid cyst in $\operatorname{IF} 4 E-5^{D 19 a}$ (B', white arrowhead). Scale bars: $100 \mu \mathrm{m}$. (D-E) Tip regions of testes stained for Soti (green, yellow arrowheads) and Adducin (magenta, red arrowheads). Soti localizes near Adducin at the distal ends of elongated spermatid cysts in wild-type (C-C') but is reduced in eIF4E-5 D19a (D-D') testes. Scale bar: 100 $\mu \mathrm{m}$. 
937 eIF4E-5;eIF4E-5 ${ }^{D 19 a}$ adult testis probed for soti mRNA (magenta; in cysts outline by dotted

938 yellow line) and stained for 3xFLAG-eIF4E-5 protein (green; red arrowheads). Scale bar: $50 \mu \mathrm{m}$.

939 (B) $3 x F L A G-e I F 4 E-5 ; e I F 4 E-5^{D 19 a}$ adult testis stained for Soti protein (magenta) and 3xFLAG-

940 eIF4E-5 protein (green) reveals varying extents of eIF4E-5 and Soti colocalization at the distal

941 ends of elongated spermatid cysts (i-iii). Boxed areas are magnified 3-fold in insets. Scale bar:

$94210 \mu \mathrm{m}$.

Fig. 6. Accumulation of axonemal dynein Kl-3 is independent of $e I F 4 E-5$. (A-F) Laserscanning confocal fluorescence micrographs. (A-C) Wild-type whole adult testes probed for $k l-3$ mRNA (magenta) and soti mRNA (green) and stained for DNA (white). $k l-3$ mRNA (cysts outlined by dotted yellow lines) does not colocalize with soti mRNA at the distal end of elongating spermatid cysts. Scale bars: $50 \mu \mathrm{m}$. (D-F) Wild-type whole adult testes probed for $\mathrm{kl}$ 3 mRNA (magenta) and stained for 3xFLAG-eIF4E-5 protein (green) and DNA (white). $k l-3$ mRNA (cysts outlined by dotted yellow lines) does not colocalize with eIF4E-5 protein (red arrowheads) at the distal ends of early or late elongating spermatid cysts. Scale bars: $50 \mu \mathrm{m}$. (G)

952 Immunoblots of whole testis extracts revealing K1-3 3xFLAG levels in the indicated genotypes 953 expressing endogenously tagged K1-3 3xFLAG. K1-3 3xFLAG protein levels are unaffected in 954 eIF4E-5 mutants.

Fig. 7. eIF4E-5 acts with Orb2 and aPKC to regulate spermatid cyst polarity. (A-D) Laserscanning confocal fluorescence micrographs of wild-type (A, C) or $\operatorname{eIF} 4 E-5^{B 8 b}(\mathrm{~B}, \mathrm{D})$ whole adult testes stained for DNA (magenta) and F-actin (green). Nuclei in elongated spermatid cysts orient towards the basal end in wild-type testes, whereas occasional clusters of nuclei orient towards the testis tip in $e I F 4 E-5^{B 8 b}$ mutants (B, D). Scale bars: $100 \mu \mathrm{m}$. (E-F) Percentage of testes that have at least one cluster of spermatid nuclei found at the tip instead of the basal end of the testes. Error bars show standard deviation based on three sets of experiments. Student $t$-tests were unpaired. (E) The percentage of testes with misoriented spermatid cysts was significantly higher in homozygous eIF4E-5 mutants as compared to wild type: $e I F 4 E-5^{B 8 a}(p<0.05)$ and $e I F 4 E-5^{B 8 b}(p<0.01)$. Number of testes scored for each genotype, from left to right: wild type = $96617 ; e I F 4 E-5^{B 8 a}=32 ; e I F 4 E-5^{B 8 b}=25$. (F) The percentage of testes with misoriented spermatid 967 cysts was significantly higher in each of the four transheterozygotes relative to their respective 
968

969

970

971

972

973

974

975

976

977

978

979

980

981

982

983

984

985

986

987

988

989

990

991

992

993

994

995

996

997

heterozygous controls: $a P K C^{06403 /+}$; $e I F 4 E-5^{B 8 a} /+$ and $a P K C^{06403} /+(p<0.05)$ and $e I F 4 E-5^{B 8 a} /+$ $(p<0.05), a P K C^{06403 /+}$; eIF4E-5 $5^{B 8 b} /+$ and $a P K C^{06403} /+(p<0.01)$ and $e I F 4 E-5^{B 8 b} /+(p<0.01)$, $o r b 2^{36} / e I F 4 E-5^{B 8 a}$ and $o r b 2^{36} /+(p<0.05)$ and $e I F 4 E-5^{B 8 a} /+(p<0.05), o r b 2^{36} / e I F 4 E-5^{B 8 b}$ and $o r b 2^{36} /+(p<0.01)$ and $e I F 4 E-5^{B 8 b} /+(p=0.01)$. Number of testes scored for each genotype, from left to right: $a P K C^{06403 /+}=30$, orb $2^{36} /+=28, e I F 4 E-5^{B 8 a} /+=31, e I F 4 E-5^{B 8 b} /+=72$, $a P K C^{06403 /+}$ eIF $4 E-5^{B 8 a} /+=145, a P K C^{06403} /+; e I F 4 E-5^{B 8 b} /+=108, o r b 2^{36} / e I F 4 E-5^{B 8 a}=117$, orb $2^{36} / e I F 4 E-5^{B 8 b}=125$.

\section{Fig. S1. Stages of spermatogenesis are organized in a spatiotemporal manner within the}

Drosophila testis. Developing germline cells are enclosed by somatic cyst cells throughout spermatogenesis. Cysts undergoing elongation and individualization have 64 spermatids but only four are shown in this schematic for simplicity.

Fig. S2. eIF4E-5 is reduced in eIF4E-5 mutants. Whole immunoblots of total testis extracts probed with anti-eIF4E-5 (A) or anti- $\alpha$-tubulin (B) reveal reduced (eIF4E-5 $5^{B 8 a}$ and $e I F 4 E-5^{B 8 b}$ ) or undetectable $\left(e I F 4 E-5^{D 19}\right)$ levels of eIF4E-5 protein while levels of tubulin loading control remained the same.

\section{Fig. S3. 3xFLAG-eIF4E-5 transgene restores formation of mature sperm and waste bags in} eIF4E-5 mutants. Phase-contrast micrographs. (A-F) 7-day old wild type (A), eIF 4E-5 $5^{B 8 a}$ (B, $e I F 4 E-5^{B 8 b}(\mathrm{C}), e I F 4 E-5^{D 19 a}(\mathrm{D}), 3 x F L A G-e I F 4 E-5 ; e I F 4 E-5^{B 8 b}(\mathrm{E})$, or 3xFLAG-eIF4E-5;eIF4E$5^{D 19 a}(\mathrm{~F})$ revealing accumulation of mature sperm in seminal vesicles in mutants carrying 3xFLAG-eIF4E-5 transgene. Scale bar: $50 \mu \mathrm{m}$. (G) 3-day old 3xFLAG-eIF4E-5;eIF4E-5 D19a testis revealing presence of waste bag (yellow arrowheads). Scale bars: $50 \mu \mathrm{m}$.

\section{Fig. S4. eIF4E-5 interacts with multiple translational regulators in yeast two-hybrid assays.} eIF4E-5 interacts with Cup, 4E-T, eIF4G-2, and 4EBP in yeast two-hybrid assays, as revealed by growth on selective medium. Empty vectors (pOAD and pOBD2) were used as negative controls. L, leucine; W, tryptophan; A, adenine; H, histidine; 3AT, 3-amino-1,2,4-triazole. 
Fig. S5. eIF4E-5 is dispensable for expression and localization of soti mRNA. (A-F) Laserscanning confocal fluorescence micrographs of wild-type (A-C) and eIF4E-5 ${ }^{D 19 a}$ (D-F) whole adult testes probed for soti mRNA (magenta) and DNA (white). Expression and localization of soti mRNA at the distal ends of elongated cysts (cysts outlined by dotted yellow lines) appear similar in wild type and eIF4E-5 mutants. Scale bars: $25 \mu \mathrm{m}$ (A, D), $50 \mu \mathrm{m}$ (B-C, E-F). (G) Laser-scanning confocal fluorescence micrographs of a wild-type whole adult testis probed for soti mRNA (magenta) and endogenous eIF4E-5 (green). soti mRNA (cysts outlined by dotted yellow lines) does not colocalize with eIF4E-5 at the distal ends of elongated spermatid cysts (red arrowheads). Scale bar: $50 \mu \mathrm{m}$. (H-J) Laser-scanning confocal fluorescence micrographs of wild-type whole adult testes probed for soti mRNA (magenta) eIF4E-5 mRNA (green) and stained for DNA (white). Expression and localization of eIF4E-5 mRNA is diffuse during early and late stages of spermatogenesis. Scale bar: $25 \mu \mathrm{m}$.

Fig. S6. eIF4E-5 levels remain reduced in $K l-3$ 3xFLAG;eIF4E-5 mutants. Immunoblots of whole testis extracts revealing eIF4E-5 levels in the indicated genotypes expressing endogenously tagged K1-3 3xFLAG. eIF4E-5 protein levels are reduced in eIF4E-5 mutants.

Table S1. Probes for RNA FISH

\begin{tabular}{|c|c|c|}
\hline Probe Target & Fluorophore & 5'-Sequence-3' \\
\hline$k l-3$, Exon 1 & Quasar® 670 & $\begin{array}{l}\text { taacattcctttctggatcc, cgcgaaacgccaaagagttt, } \\
\text { gcagcacgctttaacatgtt, ttggtcacttacactaggtc, } \\
\text { tatcgtcttctttgttggtc, cctcatttctcgaagtaact, } \\
\text { caggcttgaaatccgttgtt, aaacataccgctggtttggg, } \\
\text { atacccaacaaatctgcaca, gttactatttcctcaggatc, } \\
\text { ttgctttcatccacaatacc, accatttacattctcaacat, } \\
\text { gggacctttttcctcaaata, cgttacttatcattatggcc, } \\
\text { cggaatcagttggatatcct, tttaagcttttcctggtagc, } \\
\text { agagcgttgaatttcagtgt, actagatccgacctcaaaca, } \\
\text { gtcgatatacaacagtccac, accgaacggttatcaatcga, } \\
\text { aaatattgccacctcatcac, aagcaagagtttcgctcttc, } \\
\text { cggctttaaaacgtgatcca, caaattcggtcacagcttct, }\end{array}$ \\
\hline
\end{tabular}




\begin{tabular}{|c|c|c|}
\hline & & $\begin{array}{l}\text { tgagtttgctctttttctgc, acagttgcttcagatgattt, } \\
\text { cctttaaatagttcatgcga, tttgccatctcacaagtaat, } \\
\text { gtatttttgaactggcttca, caaccagtcgaactcgtgta, } \\
\text { gccattgctcaaaataacgt, gtataccttgaatttgtcga, } \\
\text { gtatttgtttccctctact, ctacatcgggtgtatctctt, } \\
\text { ccaattgaccaacatttgca, tatatctggccaacatacgc, } \\
\text { gtaacaaactccgttatggt, gtggttattaaatgctcggg, } \\
\text { agataatgtcaagcaatcct }\end{array}$ \\
\hline$k l-3$, Exon 14 & Quasar ${ }^{\circledR} 570$ & $\begin{array}{l}\text { gtactttgacatagccatgg, aagatttgcctttaagggca, } \\
\text { tgatatttagcctcttgcac, ctgcttcttgtagatcactt, } \\
\text { cgttttctttttgttgcagt, tttttggcctcgtctaatac, } \\
\text { aaccaccaataagagcggtt, ttcagtccatcggattttt, } \\
\text { cggtcggtctcacttttaaa, ggagaataacatctccgacc, } \\
\text { tcttgattaaatggtcccgt, atgttccactcaccaatttg, } \\
\text { ttgacagttcatctgttggt, ttttaatccacacttttccc, } \\
\text { taatcggaattcccatgcta, taactcctctgcaacatctt, } \\
\text { aaggttgtccaagcaaggat, tccaatccacttctttatca, } \\
\text { gattgggtagctttgttgta, cgcgcaaatatttcaggtgt, } \\
\text { ccacgcattgtcacagtaaa, gaacccgttcatcttctaat, } \\
\text { tttcatgtttccagtcacag, ttccttcgtagtggacaac, } \\
\text { cctcaatcactgtaacgtcg, tccttaacttcaatggcagt, } \\
\text { cagcgtttatttttgcttct, acactacctcttgtagcaac, } \\
\text { gcatcaaagcgctcaaggaa, tcctctagatttgtagcgat, } \\
\text { ttaactcaagctggcgatca, cgcaccgccttttataaata, } \\
\text { caccgaaacggaacaggagg, tataccacgtaaaccaagcc, } \\
\text { ccatacaccacgaacgaaca, agtttagtactactggctcg, } \\
\text { aatcattggcataagttccc, agctcatttttttggcgag, } \\
\text { cttggcccatagaaatagga, cgtcttctaggcaggataat, } \\
\text { tcctaagtgacagttttgca, actgtaagctctaccatgta, } \\
\text { agcaggtggttcattagtat, tttttagtccagcacgtatt, }\end{array}$ \\
\hline
\end{tabular}




\begin{tabular}{|c|c|c|}
\hline & & $\begin{array}{l}\text { tggagattgcgaatagtcca, tgcataccagtcggatgaat, } \\
\text { tcctgctccttaaaactgta, cgtttgccatgtcatcaata }\end{array}$ \\
\hline soti & Quasar ${ }^{\circledR} 570$ & $\begin{array}{l}\text { Tctcgacgaggtaatttg, tccgtgtagtacgtccat, } \\
\text { gctcatcgtacagatcgt, ccgactcgatcgattagc, } \\
\text { atcttcattcaccgcgtc, tgtccaagtcatcgccag, } \\
\text { tgctgtccatcctccaat, tgacgattgactcccagg, } \\
\text { gtccaggagtatgtccat, caacggtggctcttgagg, } \\
\text { ctccttgcgccggaaaaa, acgtggtggtccatttgg, } \\
\text { aacttcgtttcttccgcc, ggagtgggtttggtcata, } \\
\text { ctcctgactttggcatgg, ttaggaggcacatctccg, } \\
\text { attgccetcgtgacactg, atcctcgcgaacgtgacg, } \\
\text { caaagtactcgcctcgct, gggtagttctgactggtc, } \\
\text { tggcagacccataccatt, agaactgaccccaatgct }\end{array}$ \\
\hline eIF4E-5 & Quasar® 670 & $\begin{array}{l}\text { ttgcacttgtgcactggc, tgatcctgctgcttcata, } \\
\text { ctattcagctgatgttgc, ccttcgtggtgatgatga, } \\
\text { ttgctctccagtggaaca, atcgataggttctgggct, } \\
\text { gcttgtactcgacttggt, gtccacacatgctccaaa, } \\
\text { ggtgcgatcgttttccaa, catgtccttccagtgctt, } \\
\text { atctcggtaatctcgttt, aaggtctccacactgtca, } \\
\text { gtatggtacaagctccag, tccgctggggtcttgatg, } \\
\text { tcgcatccaatcttcagc, tgccettcttgaacacgg, } \\
\text { cttcatcctcccacatgg, cgaccaccettgatgttg, } \\
\text { ttactcacggtgaccagc, aattccgctttggcactc, } \\
\text { gcagtagaatgtccagcc, aattttggcccaccatca, } \\
\text { atctcgtcggagtattca, ttgatgacagctccgcag, } \\
\text { ttgttgctcttgttacgt, tttcgttactcccgttgg, } \\
\text { cgatctccagaatggcca, gtatcttcagcttctggc, } \\
\text { tttggacattgcatcgga, aaaccgatttgacgcceg }\end{array}$ \\
\hline
\end{tabular}




\section{Shao Figure 1}

A

7895K

7895.5K

7896K

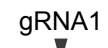

$\nabla$

gRNA2

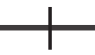

wild type ACCAAGTCGAGTACAAGCATCCTTTGGAGCATGTGT elF4E-588a ACCAAGTCGAGTACAAG-- - - - - - - CATGTGT elF4E-5 $5^{\text {B8b }}$ ACCAAGTCGAGTACAAGC---CTTTGGAGCATGTGT elF4E-5 ${ }^{\text {D19a ACCAAGTCGAGTACAAGCATC-TTTGGAGCATGTGT }}$

B

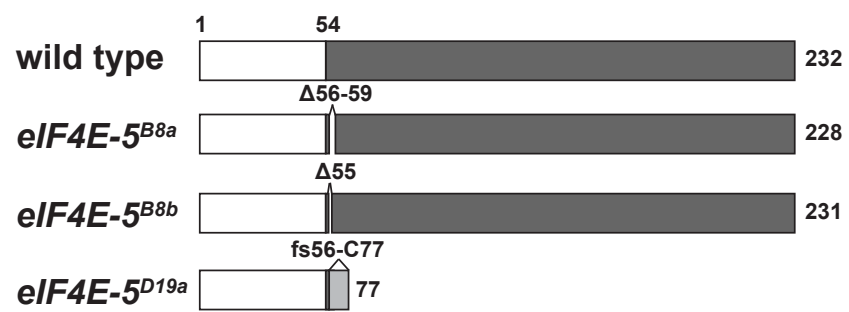

C

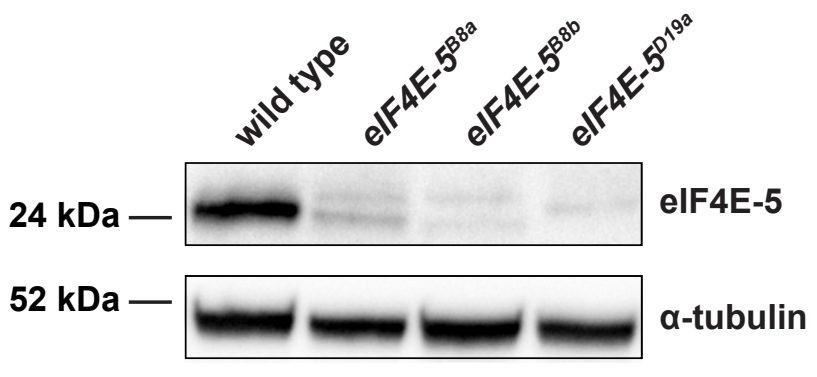

D

Genotype
wild type
elF4E-5
elF4E
elF4E-5 $5^{\text {B8b }}$

elF4E-5 $5^{B 8 a} / D f(3 L) B S C 631$ elF4E-5 $5^{B 8 b} / D f(3 L) B S C 631$ elF4E-5 $5^{D 19 a} / D f(3 L) B S C 631$ elF4E-5 ${ }^{88 a} / D f(3 L) E x e / 6279$ elF4E-5 ${ }^{B 8 b} / D f(3 L) E x e / 6279$ elF4E-5 ${ }^{D 19} / D f(3 L) E x e / 6279$ 3xFLAG-elF4E-5; elF4E-5 3XFLAG-elF4E-5; elF4E-5

\begin{tabular}{|c|} 
Male Fertile \\
\hline Yes \\
No \\
No \\
No \\
No \\
No \\
No \\
No \\
No \\
No \\
Yes \\
Yes
\end{tabular}
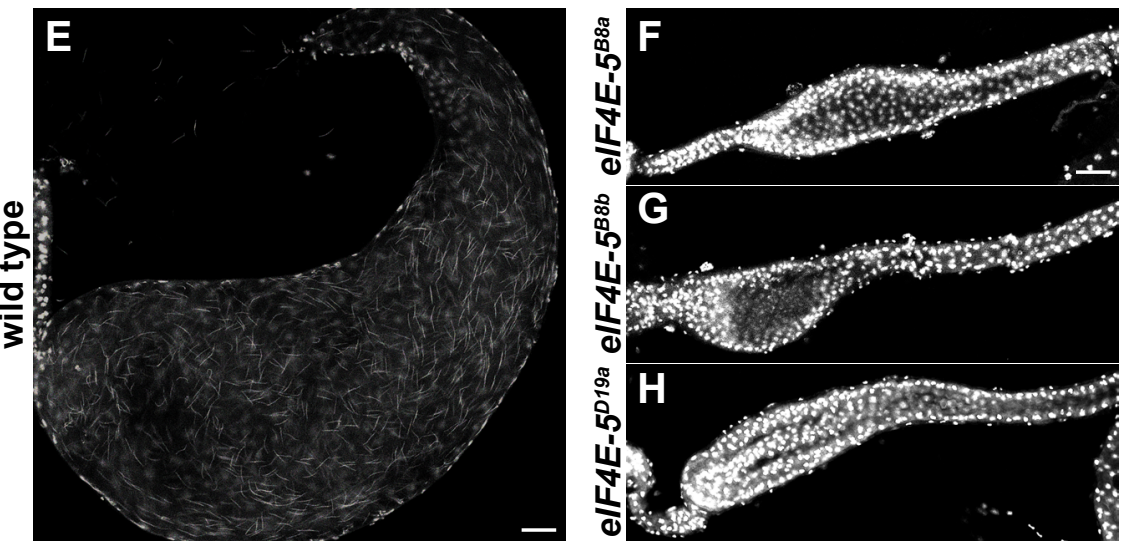
Shao Figure 3
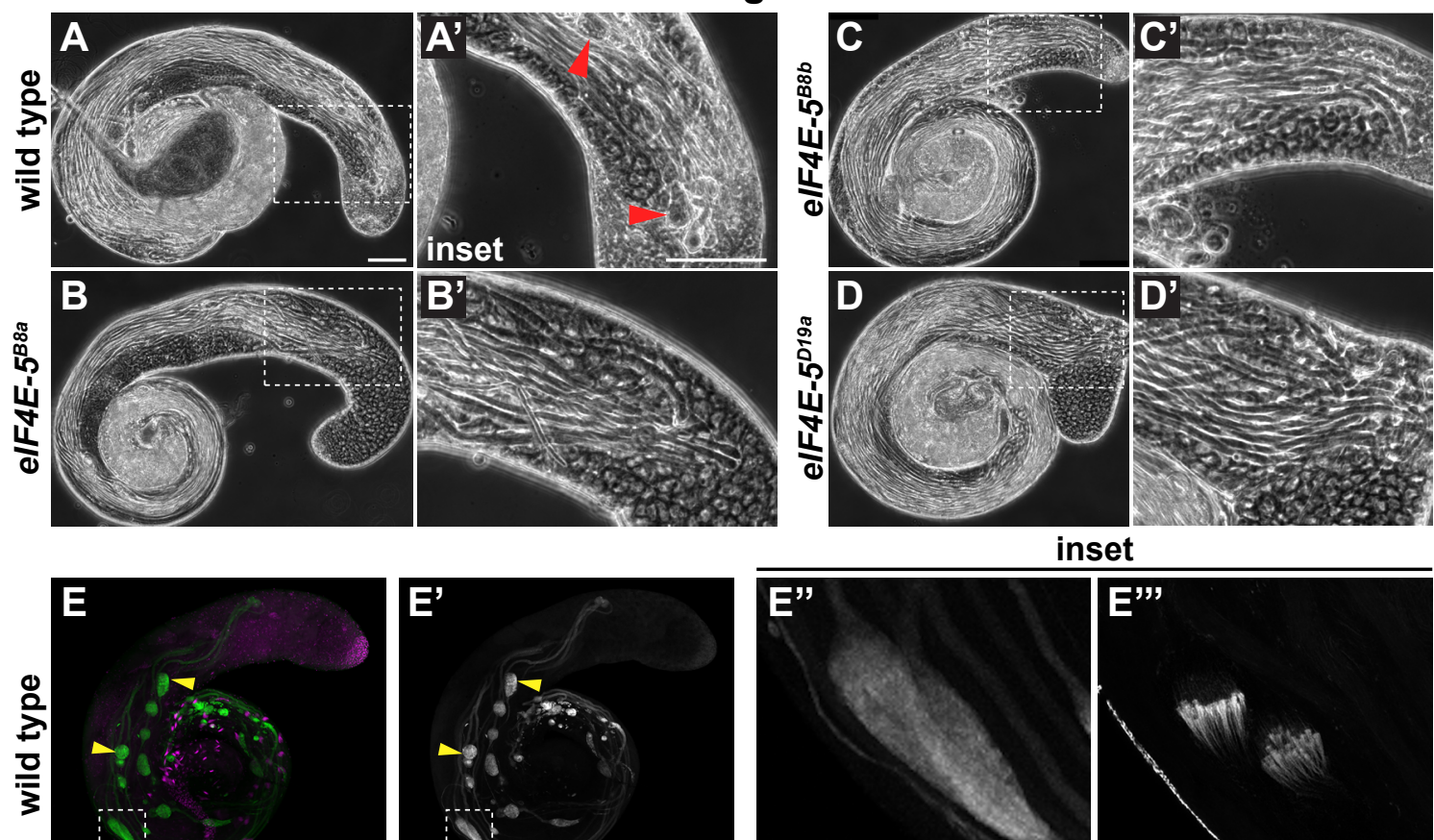

E'

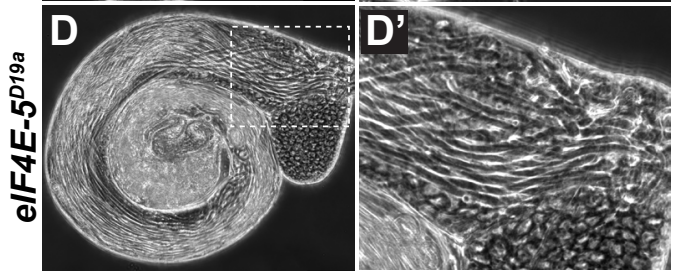

inset
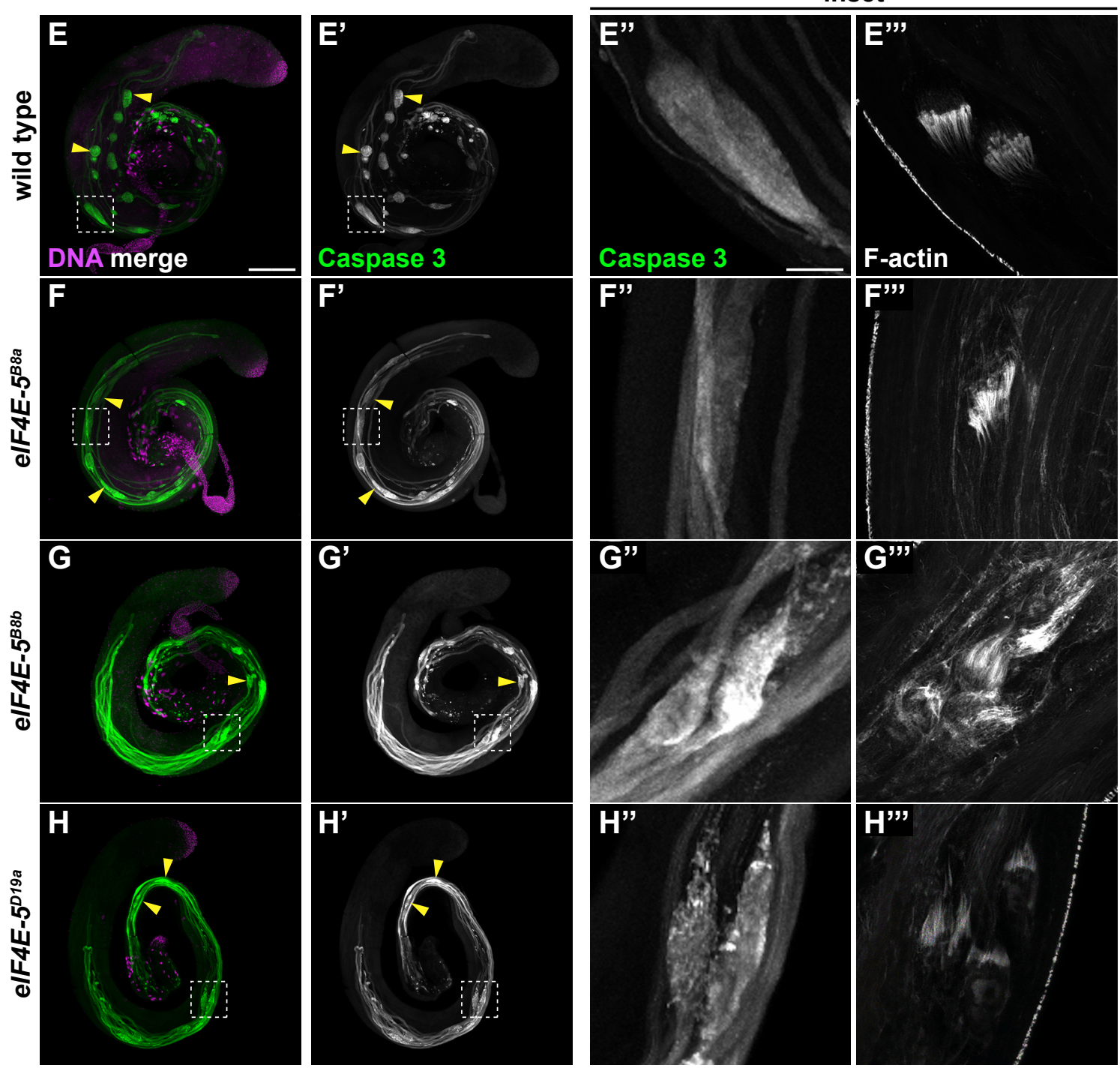


\section{Shao Figure 4}

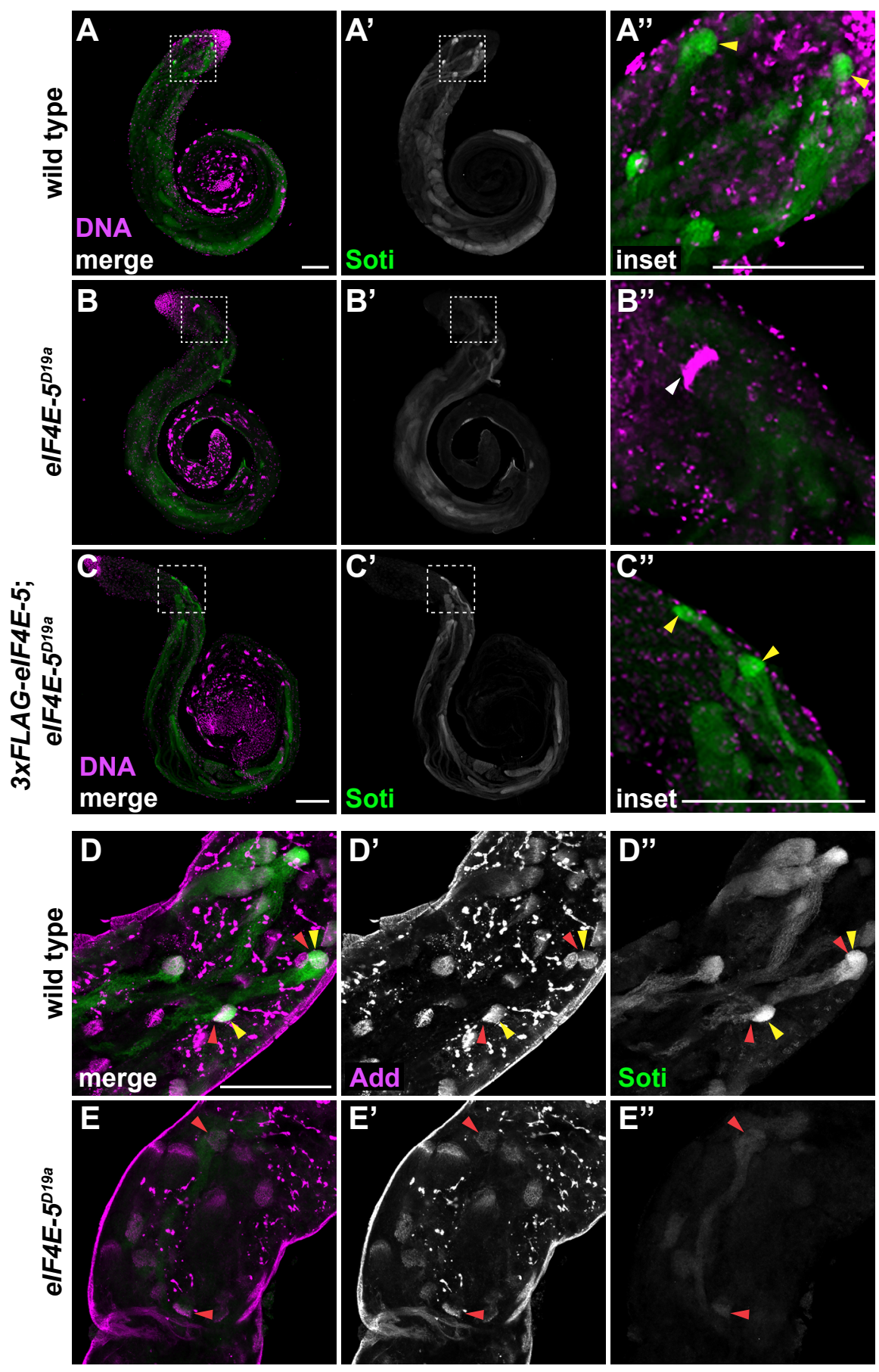


Shao Figure 5






\section{Shao Figure 7}

wild type

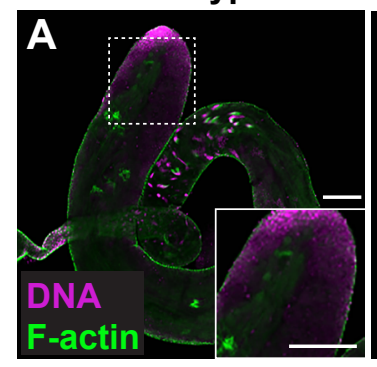

wild type

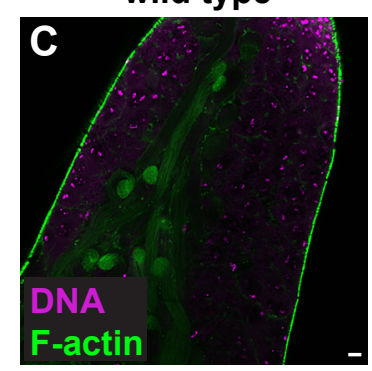

elF4E-5 $5^{88 b}$

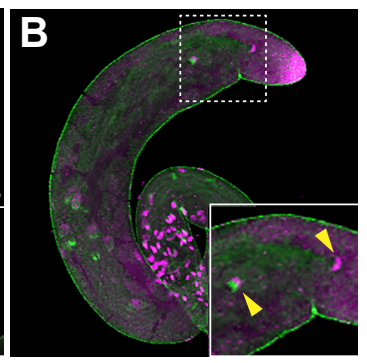

eIF4E-5 ${ }^{88 b}$

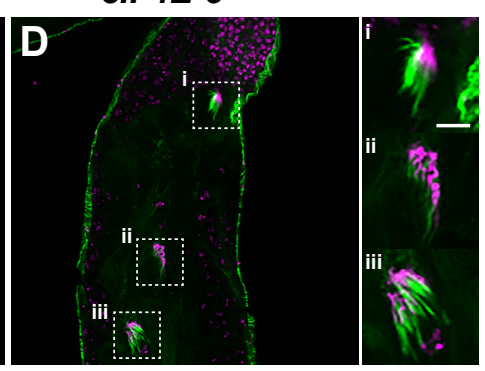

E



F

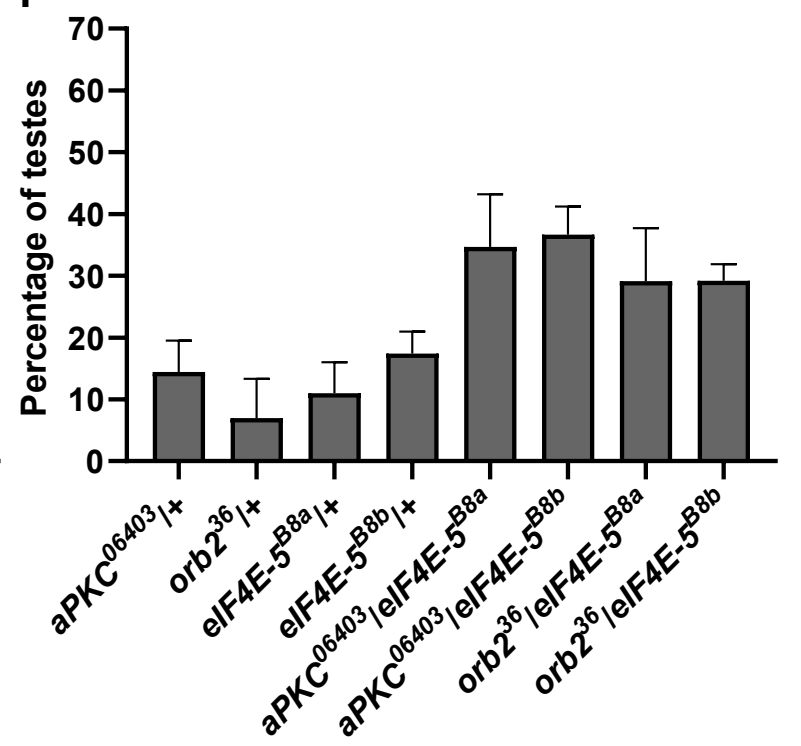




\section{Shao Figure S1}






\section{Shao Figure S2}

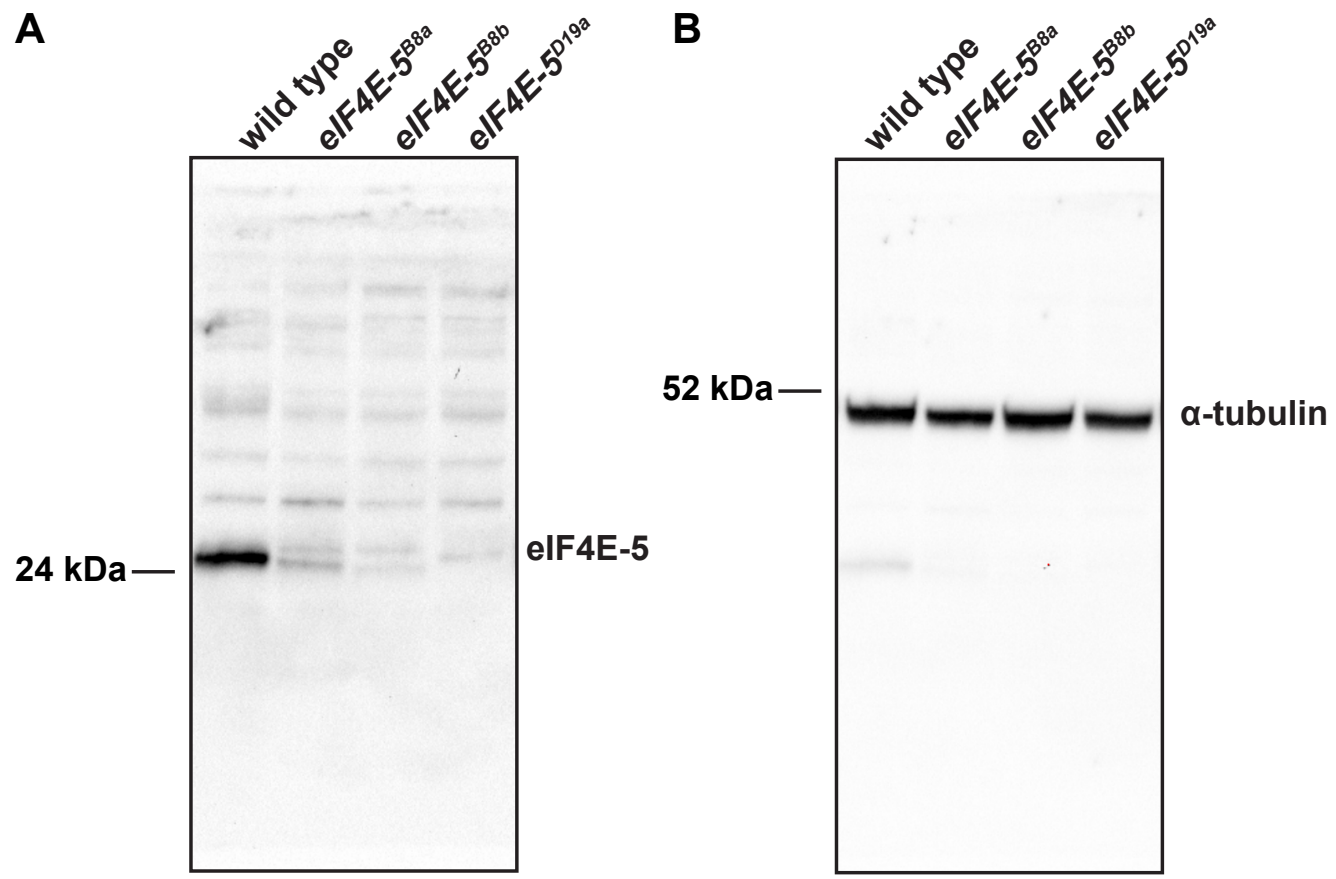




\section{Shao Figure S3}

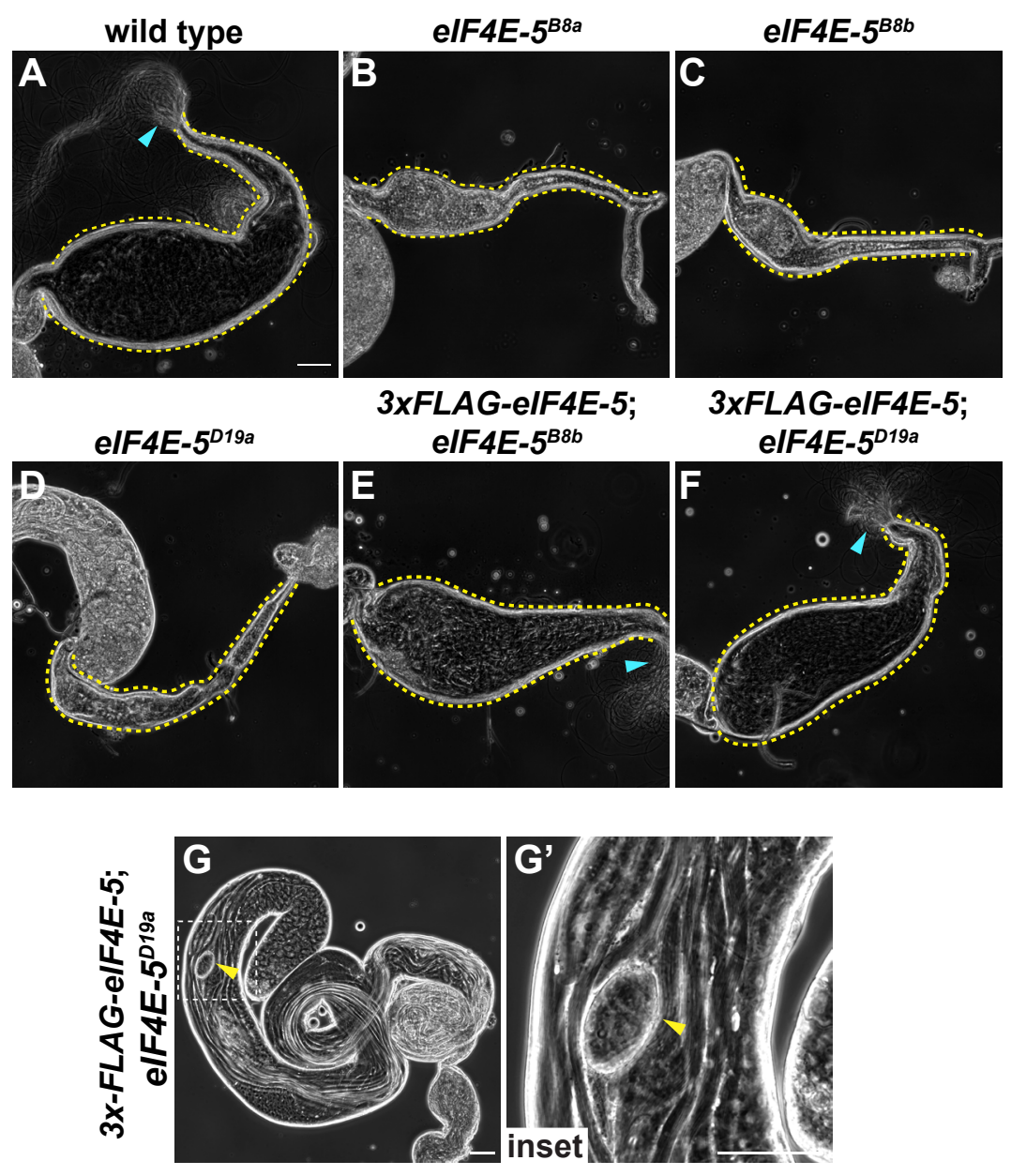




\section{Shao Figure S4}

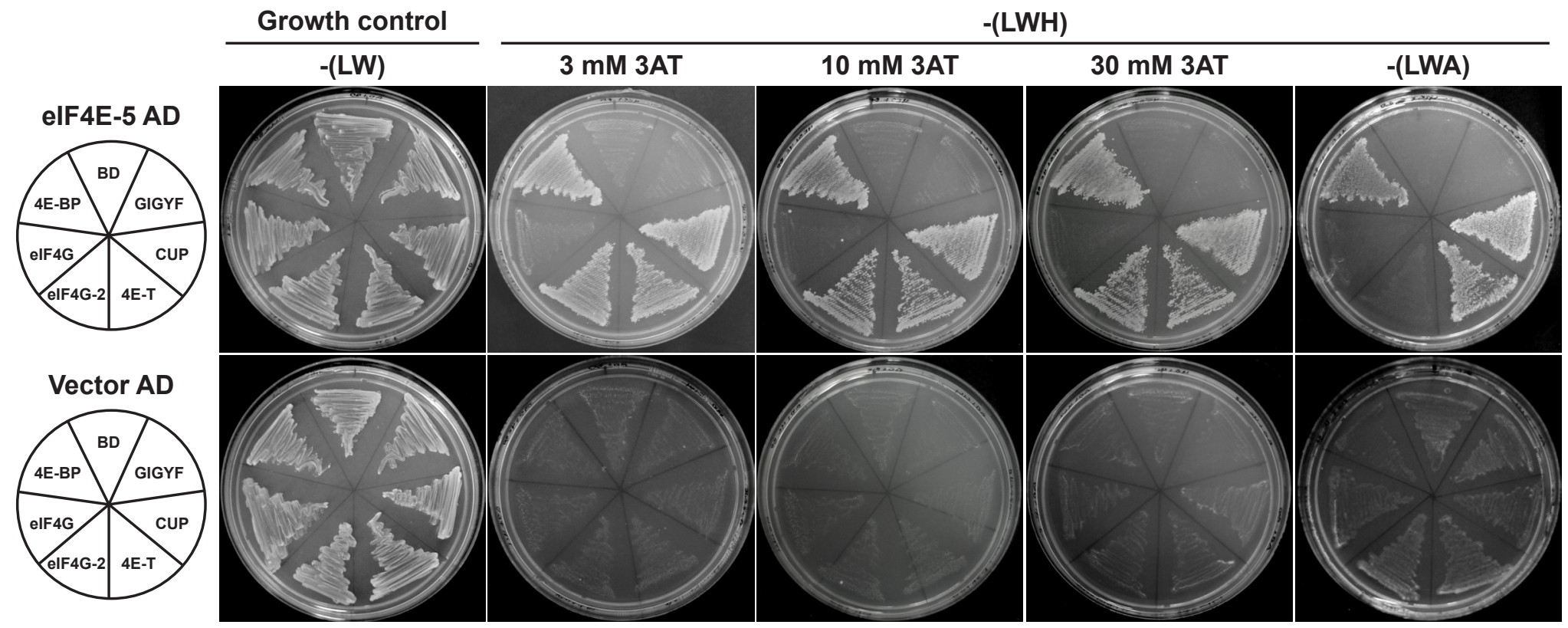




\section{Shao Figure S5}
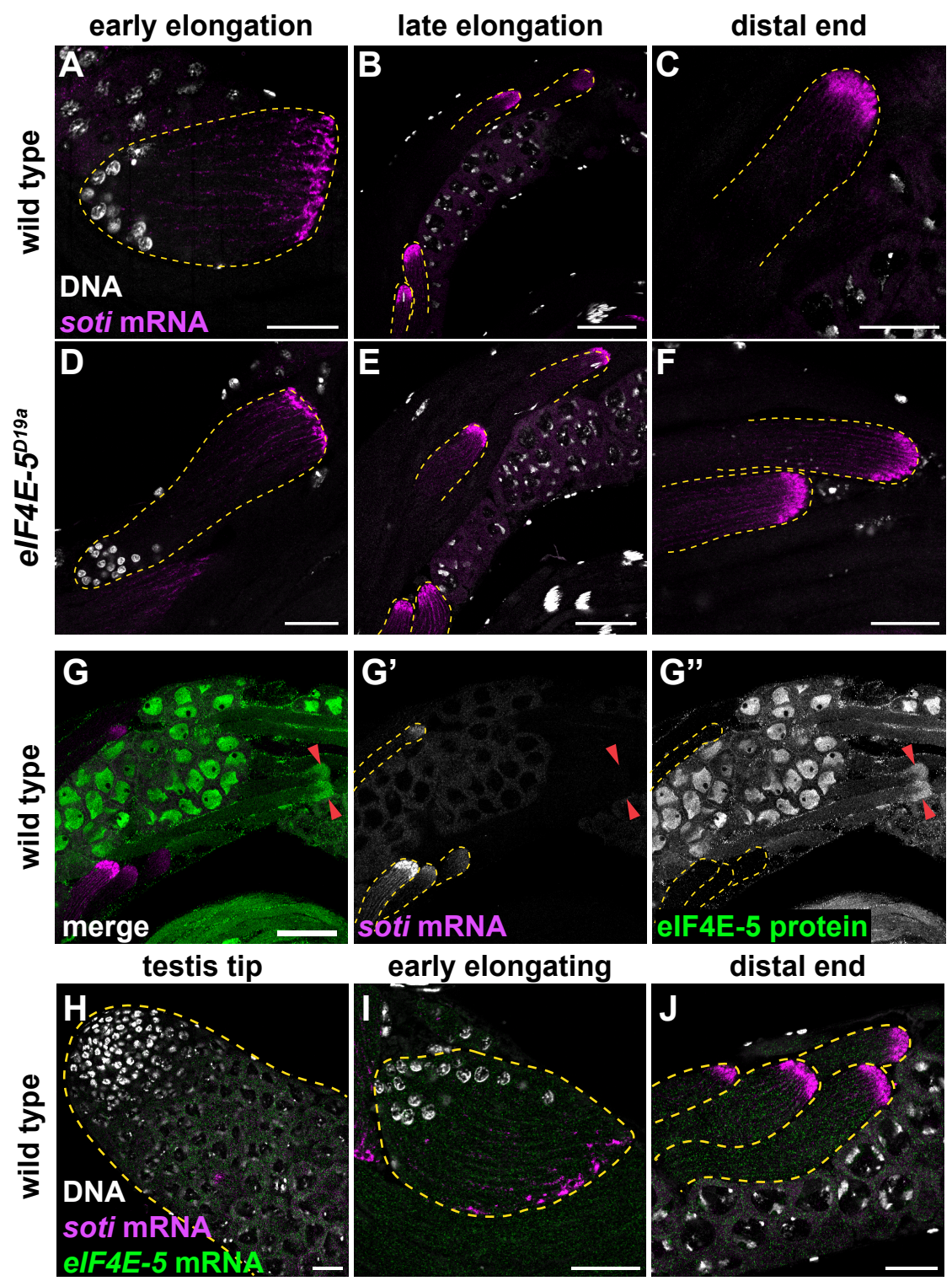

distal end




Shao Figure S6

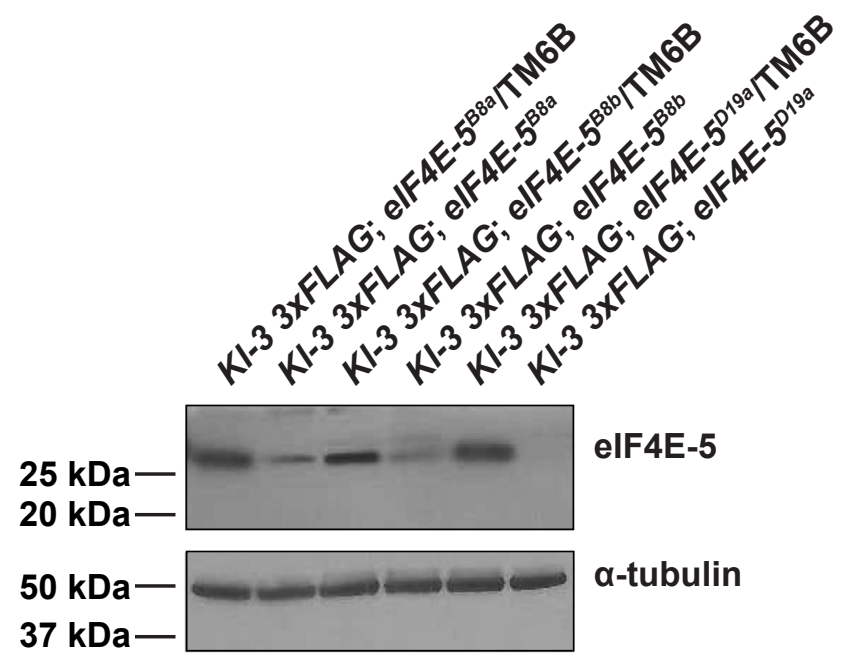

